\title{
Stromal miR-200s contribute to breast cancer cell invasion through CAF activation and ECM remodeling
}

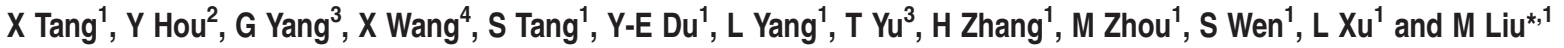

The activation of cancer-associated fibroblasts (CAFs) is a key event in tumor progression, and alternative extracellular matrix (ECM) proteins derived from CAFs induce ECM remodeling and cancer cell invasion. Here we found that miR-200 s, which are generally downregulated in activated CAFs in breast cancer tissues and in normal fibroblasts (NFs) activated by breast cancer cells, are direct mediators of NF reprogramming into CAFs and of ECM remodeling. NFs with downregulated miR-200 s displayed the traits of activated CAFs, including accelerated migration and invasion. Ectopic expression of miR-200 s in CAFs at least partially restored the phenotypes of NFs. CAF activation may be governed by the targets of miR-200 s, Fli- 1 and TCF12, which are responsible for cell development and differentiation; Fli-1 and TCF12 were obviously elevated in CAFs. Furthermore, miR-200 s and their targets influenced collagen contraction by CAFs. The upregulation of fibronectin and lysyl oxidase directly by miR-200 or indirectly through Fli-1 or TCF12 contributed to ECM remodeling, triggering the invasion and metastasis of breast cancer cells both in vitro and vivo. Thus, these data provide important and novel insights into breast CAF activation and ECM remodeling, which trigger tumor cell invasion.

Cell Death and Differentiation (2016) 23, 132-145; doi:10.1038/cdd.2015.78; published online 12 June 2015

It has been well established that a reactive microenvironment induces cancer cells to proliferate, migrate and become invasive. Cancer-associated fibroblasts (CAFs) are thought to be the main players among the cohabitating stromal cell types, and they favor tumor progression. The cancerpromoting ability of CAFs is dependent on their activation; however, this process has not been fully explored.

The extracellular matrix (ECM) is a complex mixture of structural proteins, proteoglycans and glycoproteins that exerts biochemical and mechanical effects on cells. An increasing body of evidence suggests that ECM remodeling has an important role in cell morphogenesis, ${ }^{1}$ survival, ${ }^{2}$ migration and invasion. ${ }^{3}$ CAFs can deposit certain ECM components and facilitate the directional migration and invasion of carcinoma cells through mechanotransductiontriggered architectural remodeling of the microenvironment. ${ }^{4,5}$ However, the mechanism by which activated CAFs stimulate the dysregulation of ECM proteins, thus influencing cancer cell invasion, is not well understood.

Previously, our team identified a set of dysregulated miRNAs in breast CAFs using a miRNA microarray, and it was found that the levels of miR-200 family members were noticeably suppressed, ${ }^{6}$ indicating their importance in CAF function. Whether these downregulated miR-200 s in the stroma drive the activated phenotype of CAFs as well as aberrant ECM protein expression to promote cancer cell invasion is an intriguing question.

The miR-200 s family can be functionally divided into cluster 1 (miR-200a and miR-141) and cluster 2 (miR-200b and miR-200c) according to their 'seed' region for binding to mRNA. The effects of the miR-200 s on fibrosis, epithelial cell characteristics, cell differentiation and tumor progression have been discussed. For example, miR-200b is essential for the regulation of renal fibrogenesis ${ }^{7}$ and the intestinal fibrosis of Crohn's disease. ${ }^{8}$ In aggressive carcinoma cells, the maintenance of $\mathrm{EMT}^{9}{ }^{9}$ tumor growth, ${ }^{10}$ migration, ${ }^{11}$ invasion, ${ }^{9}$ anoikis resistance ${ }^{12}$ and poor response to chemotherapy ${ }^{13}$ are enhanced by the reduced expression of miR-200 s. Furthermore, miR-200s are upregulated during mammary differentiation ${ }^{14}$ but are downregulated in breast cancer stem cells, ${ }^{15}$ and these molecules support the maintenance of pluripotent stem cells. ${ }^{16}$ These previous reports indicate that miR-200 s may have a significant role in CAF activation.

In the current work, we first determined that miR-200 s were commonly downregulated in breast CAFs, and this result was also demonstrated in normal fibroblasts (NFs) co-cultured with breast cancer cells. miR-200 s induced the conversion of NFs into CAFs by targeting Fli-1 and TCF12. Re-expression of

${ }^{1}$ Key Laboratory of Laboratory Medical Diagnostics, Chinese Ministry of Education, Chongqing Medical University, \#1 Yi-Xue-Yuan Road, Yu-zhong District, Chongqing 400016, China; ${ }^{2}$ Experimental Teaching Center of Basic Medicine Science, \#1 Yi-Xue-Yuan Road, Yu-zhong District, Chongqing Medical University, Chongqing 400016, China; ${ }^{3}$ Department of Endocrine and Breast Surgery, The First Affiliated Hospital of Chongqing Medical University, No. 1 You-Yi Road, Yu-zhong District, Chongqing 400016, China and ${ }^{4}$ Department of Orthopaedics, The Second Affiliated Hospital, Chongqing Medical University, No. 76 Linjiang Road, Yu-zhong District, Chongqing 400010, China

${ }^{*}$ Corresponding author: M Liu, Key Laboratory of Laboratory Medical Diagnostics, Chinese Ministry of Education, Chongqing Medical University. \#1 Yi-Xue-Yuan Road, Yu-zhong District, Chongqing 400016, China. Tel: +86 23 68485938; Fax: +86 23 68485239; E-mail: mliu-hncq @ hotmail.com

Abbreviations: CAFs, cancer-associated fibroblasts; NFs, normal fibroblasts; ECM, extracellular matrix; TCF12, transcription factor 12; Fli-1, Friend leukemia integration 1; FN, fibronectin; LOX, lysyl oxidase; EMT, epithelial to mesenchymal transition; IL-6, interleukin 6; TGF- $\beta 1$, transforming growth factor- $\beta 1$; ERG, v-ets avian erythroblastosis virus E26 oncogene homolog; TCF4, transcription factor 4; $\alpha$-SMA, actin, alpha 1 skeletal muscle; FAP, fibroblast activation protein, alpha; Col-I, collagen I; MMP, matrix metalloproteinase; PR, picrosirius red; CXCL12, chemokine (C-X-C motif) ligand 12 Received 01.10.14; revised 02.5.15; accepted 04.5.15; Edited by G Melino; published online 12.6.15 
miR-200 s in CAFs attenuated the activation-associated CAF phenotype. In particular, miR-200s and their targets all contributed to CAF-associated ECM remodeling through two key ECM remodeling proteins, fibronectin (FN) and lysyl oxidase, further fueling cancer cell invasion and metastasis. Therefore, our data provide new information regarding the role of CAF activation and function in the promotion of cancer cell invasion through ECM remodeling and provide a considerable amount of information that will be useful for the development of stromal therapeutic targets.

\section{Results}

miR-200 family members (including miR-200a, miR-200b, miR-200c and miR-141) are downregulated in breast CAFs compared with NFs. In our previous miRNA profile of paired CAFs and NFs using a human miRNA microarray, ${ }^{6}$ the entire miR-200 family, with the exception of miR-429, was downregulated more than threefold in CAFs (Figures 1a and b). To expand these findings, the miR-200 s were examined by quantitative real-time PCR (qRT-PCR) in stromal CAFs and NFs that were freshly isolated from 20 breast cancer patients. The miR-200 s exhibited a greater than 2.5-fold reduction in the vast majority of CAFs (Figure 1c). Next, we investigated whether the miR-200s were downregulated in response to NF activation by breast cancer cells in the tumor microenvironment. ${ }^{17}$ Intriguingly, NFs grown in a Boyden chamber exhibited a slight decrease in miR-200 expression after individual exposure to four breast cancer cell lines (BT-474, MCF-7, MDA-MB-453 and MDA-MB-468) in six-well plates for 15 days and exhibited significantly reduced miR-200 expression after exposure for another 15 days (Figure 1d). In addition, signature CAF characteristics were acquired in these activated NFs, such as greater cytoplasmic actin polymerization, CAF biomarker expression and migration (Supplementary Figures S1a and c). These findings were further confirmed using the inverse co-culture system in which the NFs were seeded first and the breast cancer cells were seeded afterward in a Boyden chamber. Co-culture was performed for 30 days (Supplementary Figures S2a and d). Reduced levels of miR-200 s were also detected in NFs cocultured with gastric cancer cells (SGC-7901 and MGC-803; Supplementary Figure S3a). It has been reported that miR-200s could be downregulated by paracrine signaling molecules, such as IL-6 (ref 18)and transforming growth factor- $\beta$ (TGF- $\beta 1){ }^{19}$ Interestingly, higher levels of IL-6 and TGF- $\beta 1$ (Supplementary Figure S3b) were detected in the supernatant from the breast cancer cell co-culture system compared with supernatants from NFs alone or from the MCF10A cell co-culture system. TGF- $\beta 1$, but not IL-6 (Supplementary Figures S3c and d), was expressed by the breast cancer cell lines, and both SGC-7901 and MGC-803 cells have the ability to express IL-6 and TGF- $\beta 1$ (Supplementary Figure S3e). Interestingly, an elevated IL-6 level was detected in NFs after co-culture with conditioned media from cancer cells for 21 days (Supplementary Figure S3f). In addition, when NFs were cultured with $3 \mathrm{ng} / \mathrm{ml}$ TGF$\beta 1$, increased IL-6 expression and decreased expression of miR-200 s were observed (Supplementary Figure S3g).
Collectively, these data raise the possibility that the universal downregulation of miR-200 s in the breast cancer microenvironment may have a role in activating CAFs.

Fli-1 and TCF12, which are associated with cellular differentiation, are directly regulated by miR-200 s. Reprogramming NFs into CAFs represents a cellular differentiation process. We next investigated which of the targets that are directly regulated by miR-200 s are involved in CAF activation. Using Target Scan v6.2, miRanda and DIANA-microT as well as mRNA microarray data previously reported by our team, ${ }^{20} 13$ transcription factors that may be involved in cellular differentiation were identified (Supplementary Table s1). Fli-1, TCF12, ERG and TCF4 were upregulated nearly twofold in CAFs compared with NFs (Supplementary Figure S4a). In a luciferase assay, the 3'UTR of TCF12 was suppressed by miR-141, and the 3'-UTR of Fli-1 was repressed by miR-200c (Supplementary Figures S4b and c). Mutation of the binding sites in the $3^{\prime}-U T R S$ blocked the responsiveness of these genes to ectopic miR-141 or miR-200c in 293 T cells (Supplementary Figure S4d) and to endogenous miR-141/200c in CAFs (Figure 2a). The mRNA and protein levels of Fli-1 and TCF12 were decreased in CAFs after rescuing the expression of miR-200c and miR-141, respectively, and these levels were increased in NFs after knocking down miR-200c and miR-141 expression (Figures $2 b-d$ ), respectively. Thus, these data indicate that endogenous $\mathrm{Fli}-1$ is directly regulated by miR-200c and that TCF12 is directly targeted by miR-141 in CAFs.

Increased Fli-1 and TCF12 in activated NFs and in the breast cancer stromal microenvironment predict a worse outcome for breast cancer patients. The elevated expression of Fli-1 and TCF12 was first confirmed in three pairs of randomly selected NFs and CAFs (Figure 3a), and these proteins were localized to the nucleus (Supplementary Figure S5a). Consistent with the finding of gradually decreased miR-200 s in activated NFs after co-culture with breast cancer cells, increased mRNA expression levels of Fli-1 and TCF12 were detected (Supplementary Figure S5b), as were increases in total and nuclear (Figure $3 b$ ) protein levels; the nuclear localization of Fli-1 and TCF12 in activated NFs was also confirmed by immunofluorescence (IF) analysis (Supplementary Figure S5c). Stromal Fli-1 and TCF12 expression was further confirmed by immunohistochemistry (IHC) analysis of breast tumor tissues; there was negative or weak staining for Fli-1 and TCF12 in normal breast tissues and moderate or strong staining for these proteins in the nuclei of stromal cells in cases of ductal carcinoma in situ and invasive cancer (Figures $3 c$ and $4 d$ ). Interestingly, in $\mathrm{ER}^{-}$ breast tumor tissues, the stromal region had stronger TCF12 staining compared with that in $\mathrm{ER}^{+}$breast tumor tissues, and this staining pattern correlated with a poor prognosis (Figure $3 e$ ) by Kaplan-Meier survival analysis and log-rank tests using the KM plots database..$^{21}$ There was a trend suggesting that high Fli-1 expression correlated with a poor prognosis; however, the $P$-value was 0.084 (Supplementary Figure S5d). 

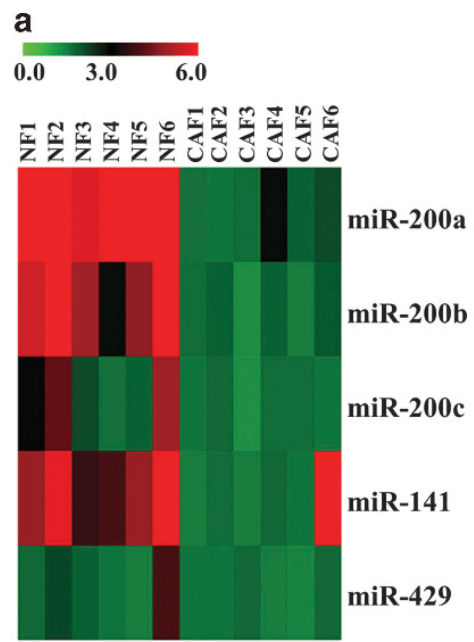

b

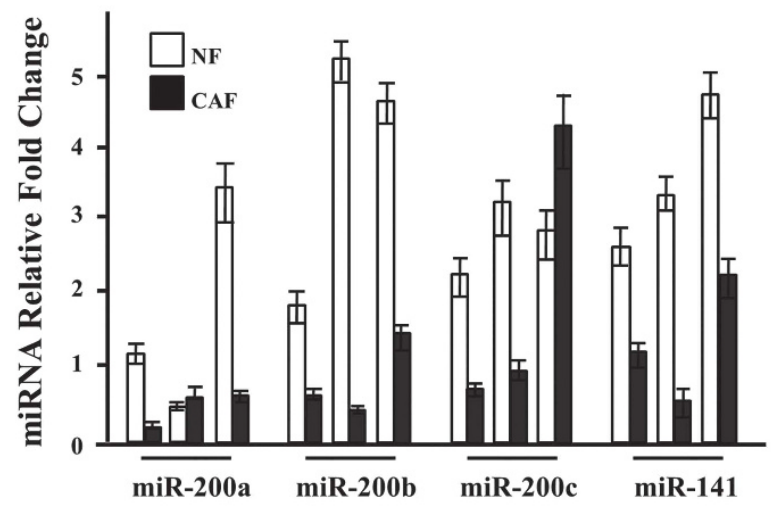

c
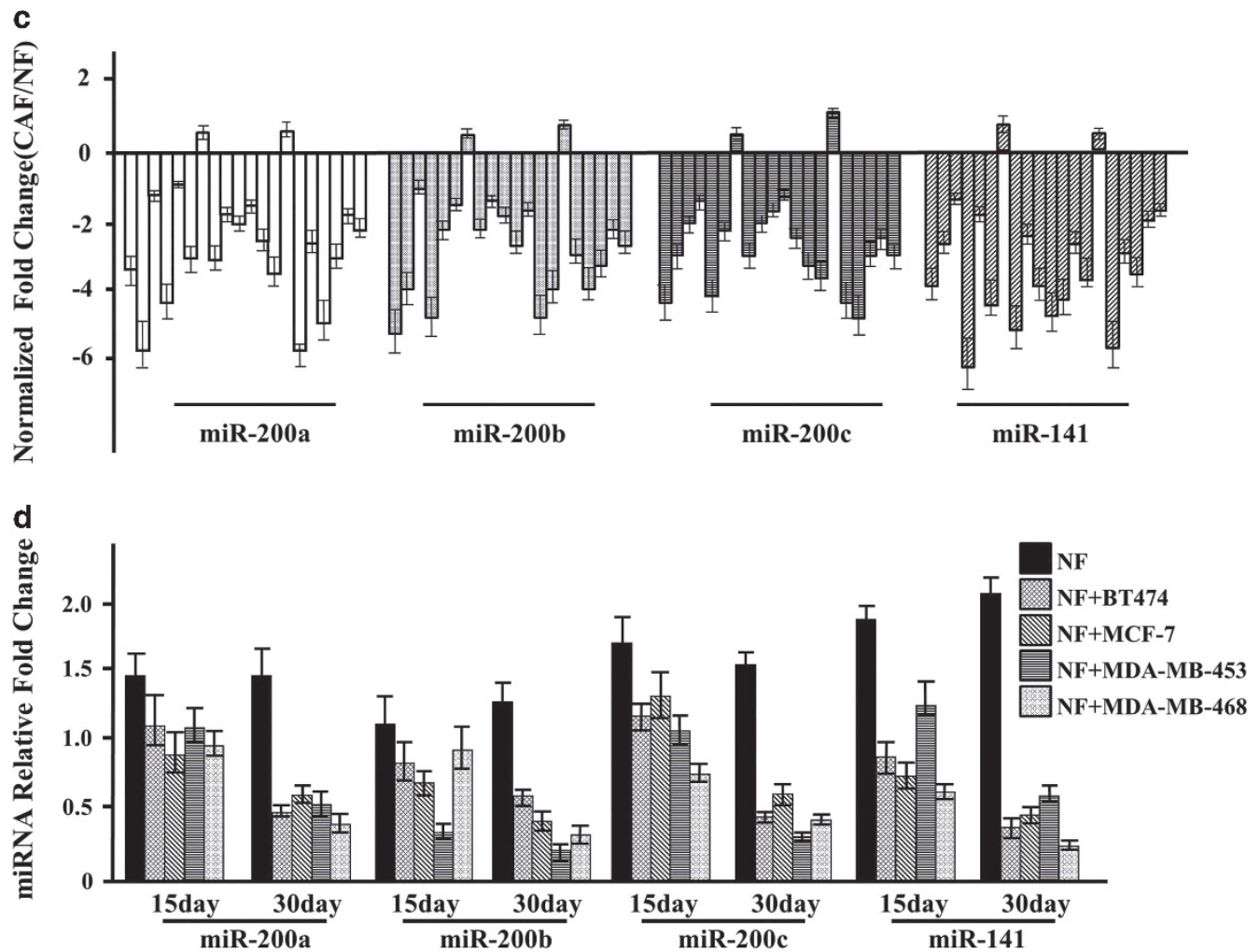

Figure 1 Expression of the miR-200 $\mathrm{s}$ is downregulated in CAFs. (a) Heat map illustrating hierarchical clustering of altered miR-200 family members in CAFs and paired NFs. (b) The expression of miR-200 s identified by miRNA array was confirmed using qRT-PCR in paired NFs and CAFs from three breast cancer patients. U6 was used as an internal control. The relative expression levels are shown as the fold change of CAFs compared with NFs $(n=3)$. (c) The expression of the miR-200 family was evaluated by qRT-PCR in 20 paired NF and CAF samples that were freshly isolated from breast carcinoma tissues. U6 was used as an internal control. The data are shown as the normalized fold change of CAFs compared with NFs $(n=3)$. (d) The expression of the miR-200 family was examined by qRT-PCR in NFs co-cultured with four different breast cancer cell lines (BT474, MCF-7, MDA-MB-453 and MDA-MB-468) for 15 or 30 days. U6 was used as an internal control. The data are shown as the normalized fold change of co-cultured NFs compared to NFs alone $(n=3)$

miR-200 s and their targets promote CAF activation. To determine whether miR-200 s and their key targets affect CAF activation, engineered CAFs that stably expressed miR-200 s and NFs with loss of function of miR-200s were established (Supplementary Figures S6a and b). The expression of the CAF marker $a$-SMA was attenuated in CAFs by both miR-200 clusters, whereas FAP expression was slightly decreased by functional cluster 2 (Figure 4a). Similarly, $a$-SMA expression in NFs increased after knocking down miR-200 functional cluster 1 or cluster 2 using shRNA, and FAP expression was only upregulated by functional cluster 2 (Figure 4b), suggesting that the miR-200 s may be essential for CAF function or activation. Inhibiting Fli-1 or TCF12 in CAFs using shRNA (Supplementary Figure S6c) 

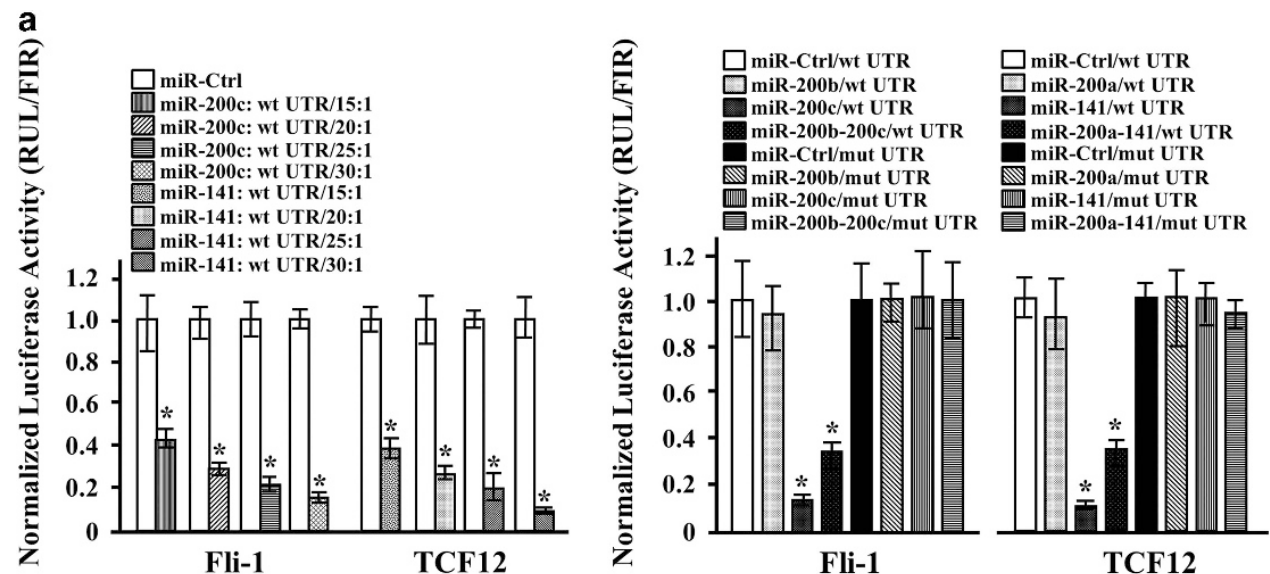

b

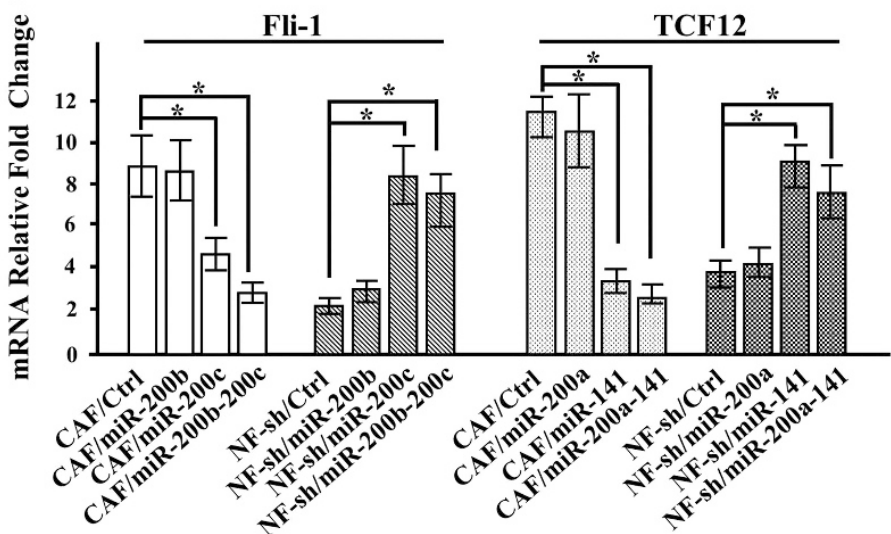

C

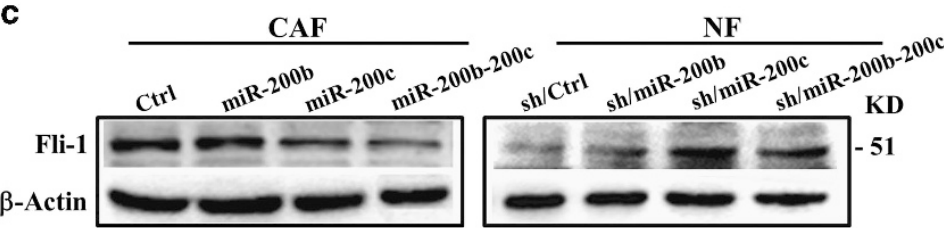

d

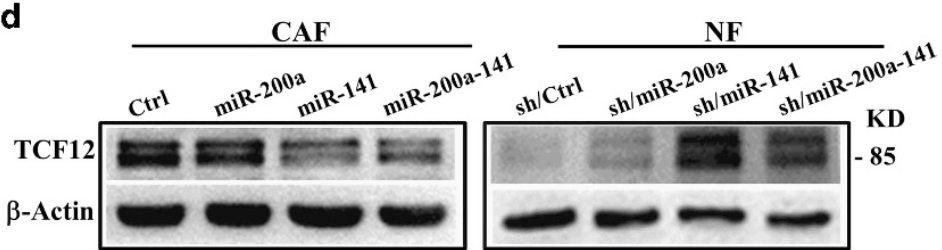

Figure 2 TCF12 and Fli-1 are directly regulated by miR-200 s. (a) Luciferase activity of CAFs co-transfected with different ratios of the indicated miR-200 s or control vector TCF12 and Fli-1 reporters driven by wild-type 3'-UTRs (left panel) and TCF12 and Fli-1 reporters driven by 3'-UTRs with wild-type or mutated miR-200 s-binding sites (right panel) are shown. miR-Ctrl, non-miRNA-expressing control; wt UTR, wild-type 3'-UTR of TCF12 or Fli-1; mut UTR, mutant-binding sites for miR-200 s in the target mRNA. All the luciferase reporter assay results were normalized to Renilla luciferase, and the data are presented as the mean \pm S.D. $\left(n=3\right.$; ${ }^{*} P<0.05$, ANOVA followed by the StudentNewman-Keuls test). (b) Lipofectamine 2000 was used to transiently transfect the miR-200 s mimics into CAFs or siRNA into NFs. Fli- 1 and TCF12 expression was examined by qRT-PCR. $\beta$-Actin was used as an internal control. The data are presented as the mean \pm S.D. ( $n=3 ;{ }^{*} P<0.05$, Student's t-test). (c and d) Western blot analysis of endogenous Fli-1 (c) and TCF12 (d) expression in the indicated fibroblasts. $\beta$-Actin was used as a loading control

decreased $a$-SMA protein levels, and ectopic expression of Fli-1 or TCF12 in NFs (Supplementary Figure S6d) increased $a$-SMA protein levels. However, FAP was only affected by Fli1, the miR-200c target (Figures $4 c$ and d). Accordingly, the ability of fibroblasts to invade into a matrix or migrate was impaired by overexpressing miR-200 s and promoted by silencing miR-200 s, as measured via transwell (Figure 4e) or wound-healing (Supplementary Figure S7a and b) assays. These results were supported by knocking down Fli-1 or TCF12 in CAFs and overexpressing Fli-1 or TCF12 in NFs (Figure $4 \mathrm{f}$ and Supplementary Figures S8a and b).

Furthermore, $a$-SMA and/or FAP expression was partially recovered by rescuing Fli-1 in CAF/miR-200c cells and TCF12 in CAF/miR-141 cells (Supplementary Figure s9a and 9b, left 
a

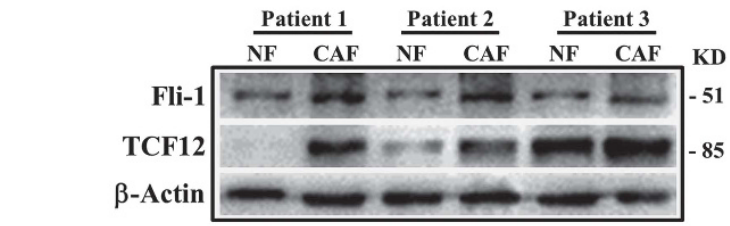

b
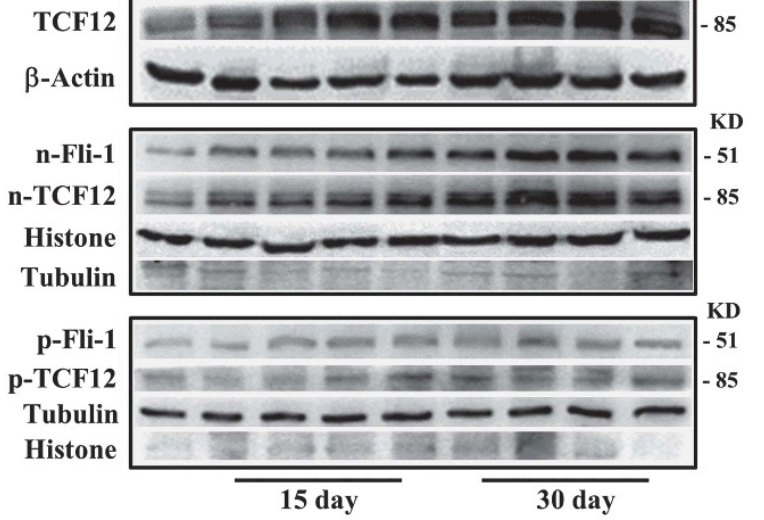

e

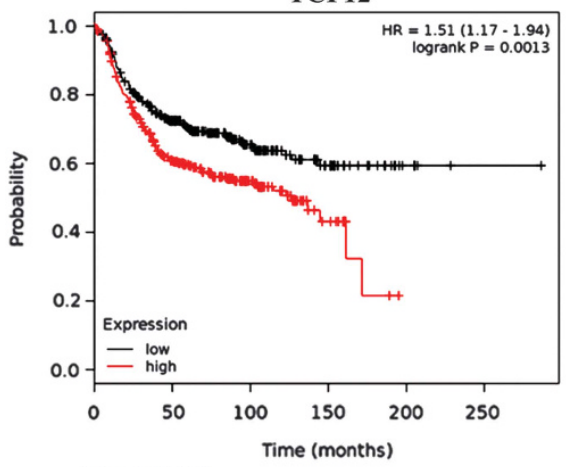

Number at risk

$\begin{array}{llllll}\text { low } 322 & 186 & 77 & 31 & 6 & 1\end{array}$
C

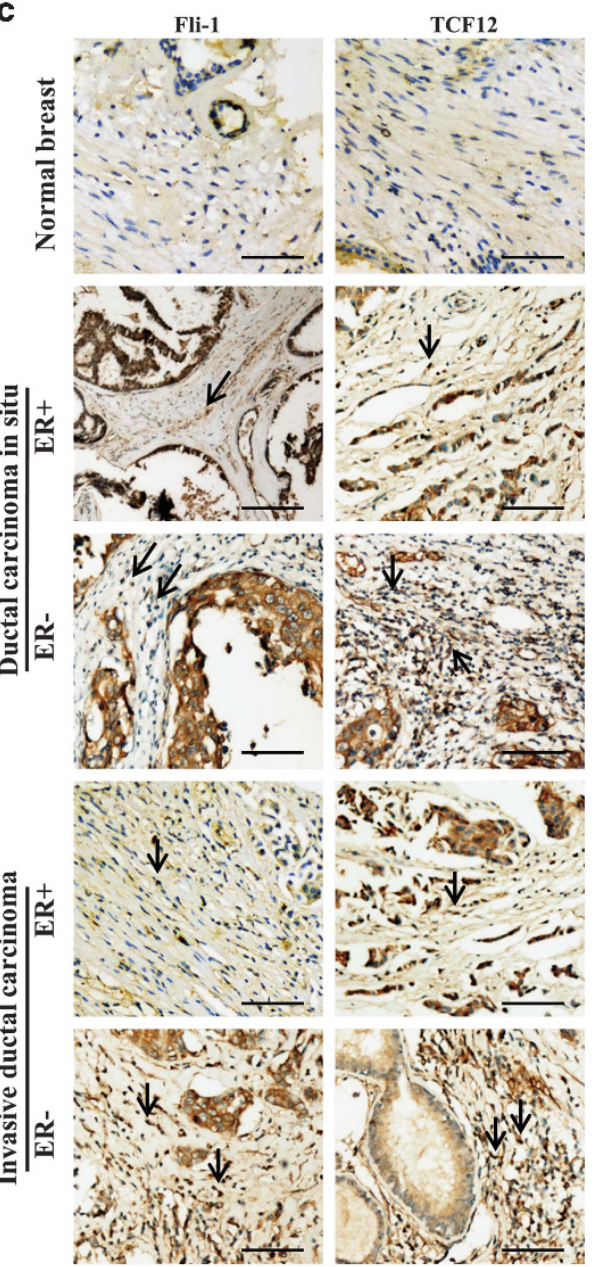

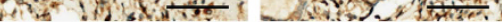

Figure 3 Fli-1 and TCF12 expression levels are upregulated in breast tumor CAFs. (a and $\mathbf{b})$ Western blot analysis of (a) endogenous Fli-1 and TCF12 protein expression in paired NFs and CAFs from three random patients or (b) total, nuclear and cytoplasmic Fli-1 and TCF12 protein expression in NFs co-cultured with four different breast cancer cell lines (BT474, MCF-7, MDA-MB-453 and MDA-MB-468) for 15 or 30 days. $\beta$-Actin, histone or tubulin was used as a loading control. (c) IHC staining for Fli-1 and TCF12 expression in breast specimens. The arrows indicate distinctly stained fibroblasts in the representative tumor tissues. Scale bars, $200 \mu \mathrm{m}$. (d) Summary of IHC staining for normal, ductal carcinoma in situ and invasive ductal breast carcinoma samples. The nuclear staining intensities were categorized as low (+), medium (++), or high (+++) based on observations of $80 \%$ of the cell population. NG, not graded. (e) The Kaplan-Meier survival analysis revealed an association between poor prognosis in ER" breast cancer patients and TCF12 expression

panel). Silencing Fli-1 and TCF12 expression again by shRNA in NF-sh/miR-200c and NF-sh/miR-141 cells decreased $a$ SMA and/or FAP expression (Supplementary Figures S9a and b, right panel). Fli-1 and TCF12 compromised miR-200c- and miR-141-mediated invasion (Supplementary Figures S9c and d) and migration (Supplementary Figures S10a and b). These data demonstrate that CAF activation can be induced by miR-200 s or their targets and that Fli-1 and TCF12 are functionally relevant effectors of miR-200c and miR-141 in CAF activation.

miR-200 $s$ and their targets Fli-1 and TCF12 are involved in ECM remodeling. CAFs have been suggested to promote cancer cell progression by secreting cytokines and chemokines or creating a distinctive ECM. ${ }^{22}$ We first sought to determine whether stromal miR-200 s could influence breast 
a

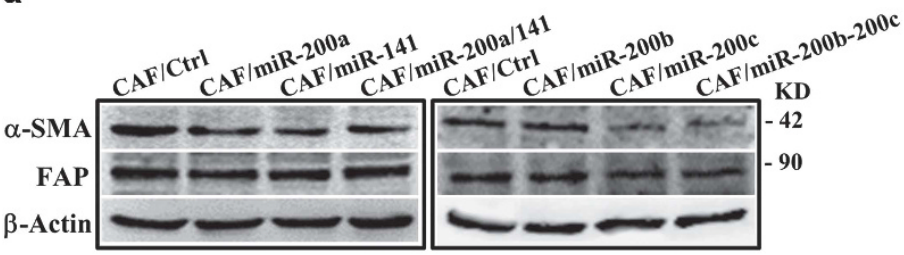

b

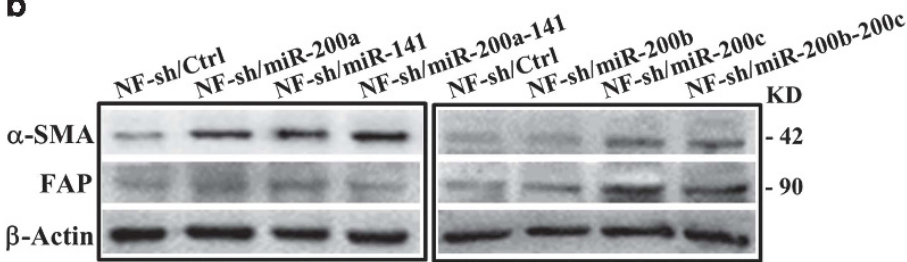

e
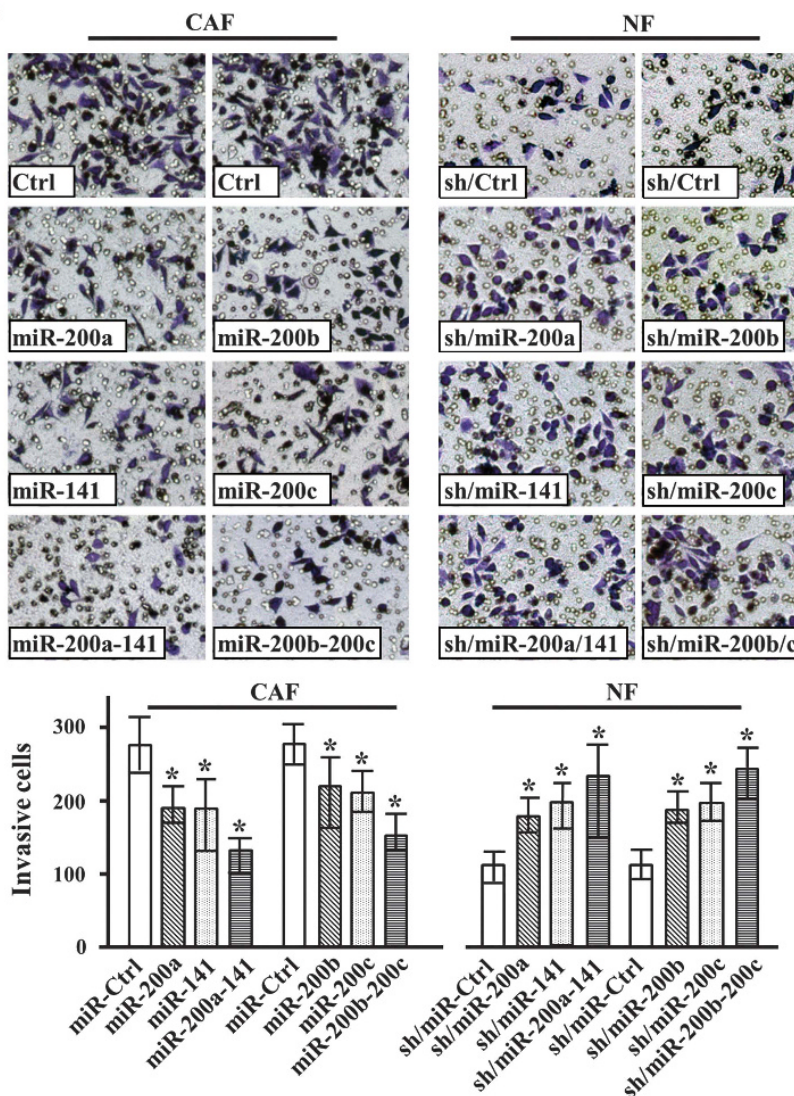

C
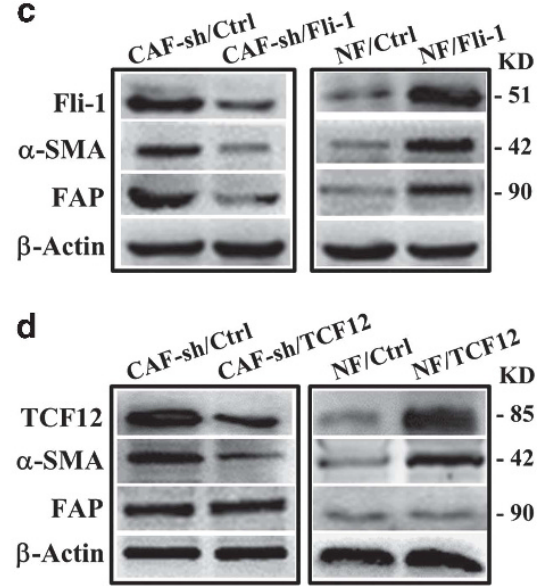

f
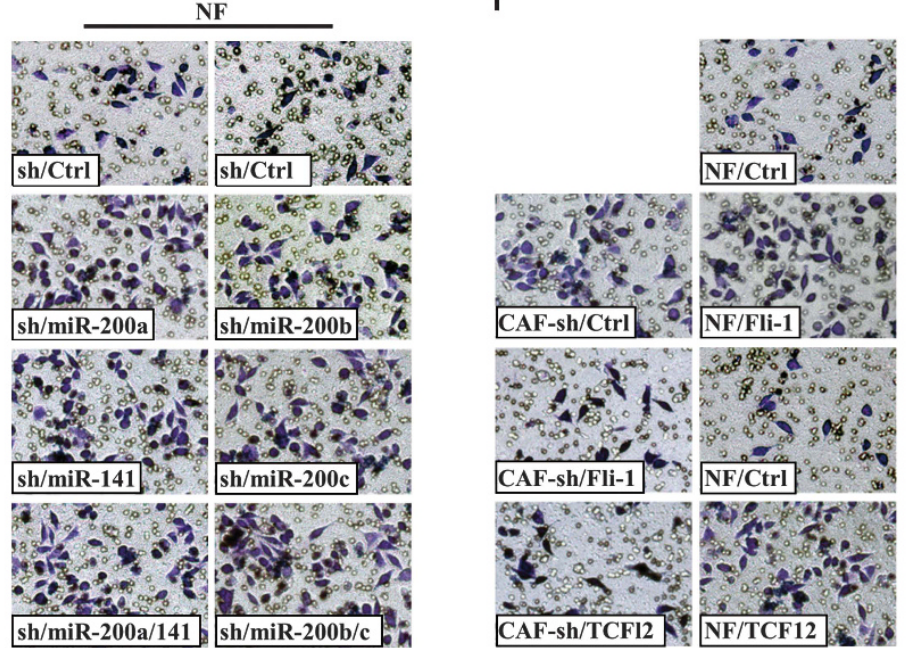

NF/Ctrl

$\square$ CAF-sh/Ctrl NF/Fli-1

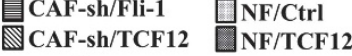

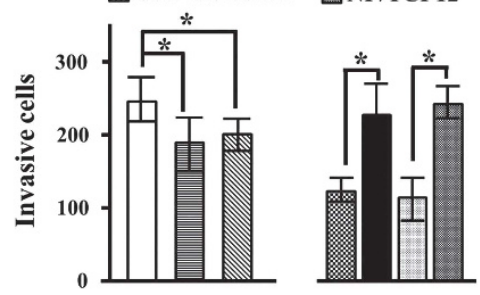

Figure 4 Decreased expression of miR-200 s and high target gene expression promote the activation of NFs to form CAFs. (a-d) Western blot analysis of $\alpha$-SMA and FAP expression in the indicated engineered CAFs or NFs. $\beta$-Actin was used as a loading control. (e) Transwell chamber analysis of the invasion of CAFs transfected with miR-200 $\mathrm{s}$ vectors or of NFs transfected with shRNAs targeting the miR-200 s. The data are presented as the mean \pm S.D. $\left(n=3 ;{ }^{*} P<0.05\right.$, ANOVA followed by the Student-NewmanKeuls test). (f) Transwell chamber analysis of the invasion of CAFs transfected with shRNAs targeting Fli-1 or TCF12 and of NFs transfected with Fli-1 or TCF12 vectors. The data are presented as the mean \pm S.D. $\left(n=3 ;{ }^{*} P<0.05\right.$, Student's t-test $)$

tumor cell migration or invasion through paracrine signaling. In a transwell co-culture system, MDA-MB-231 cell migration was less affected by exposure to CAF/miR-200 s cells than exposure to CAF/control cells (Supplementary Figure S11a), indicating a less significant impact of miR-200 s-engineered CAFs on tumor cell migration via secreted factors. Next, we questioned whether miR-200 s-induced CAF activation is associated with increased strength and stiffness of the ECM. Indeed, highly aligned and organized collagen fibers were detected in stromal breast tumor tissues (Supplementary Figure S11b). Parental CAFs had a greater ability to remodel the ECM than CAFs expressing miR-200s (Figure 5a). Conversely, the weak ability of parental NFs to remodel the ECM was enhanced by shRNA-mediated knockdown of 

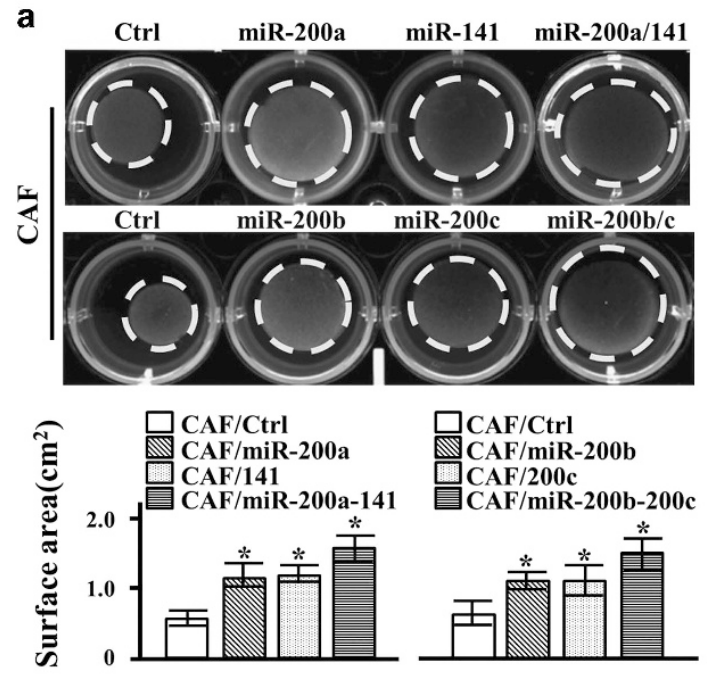

C
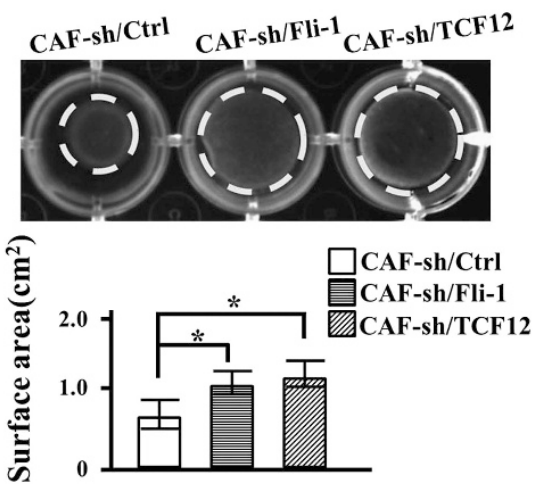

e

$\mathrm{CAF} / \mathrm{Ctrl} \quad \mathrm{CAF} / \mathrm{miR}-200 \mathrm{c} \quad \mathrm{CAF} / \mathrm{Ctrl} \quad \mathrm{CAF} / \mathrm{mi}-\mathrm{R} 200 \mathrm{c}$

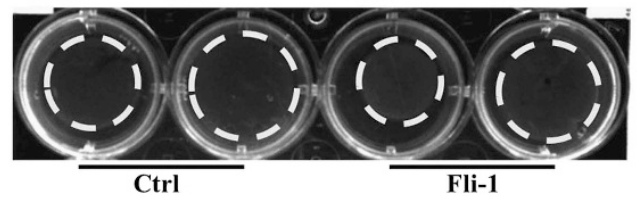

CAF/Ctrl CAF/miR-141 CAF/Ctrl CAF/miR-141
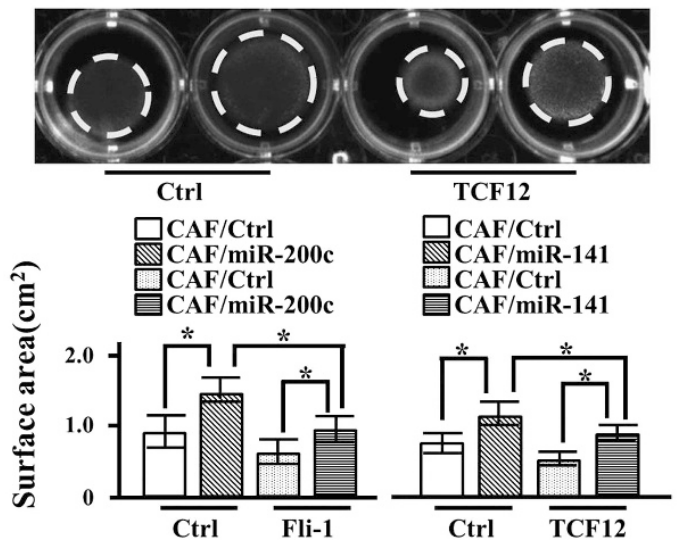

b
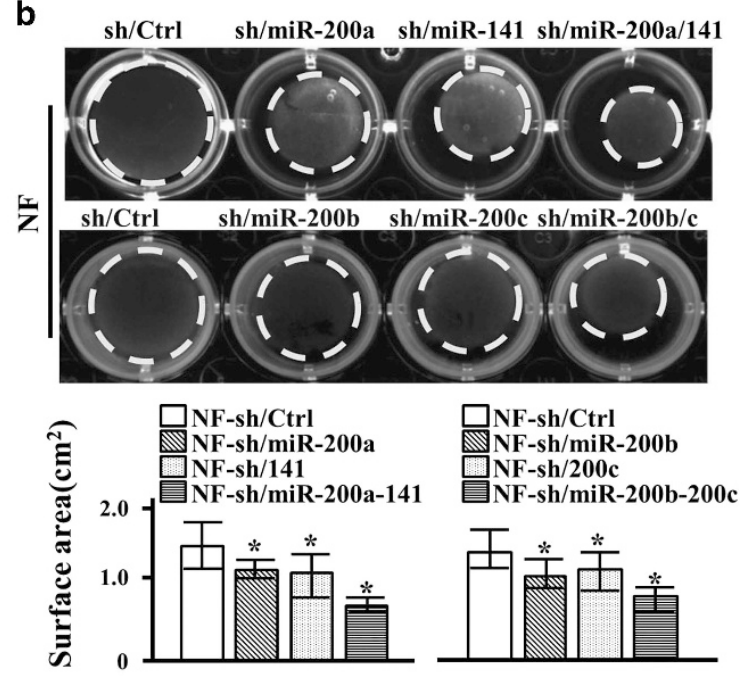

d
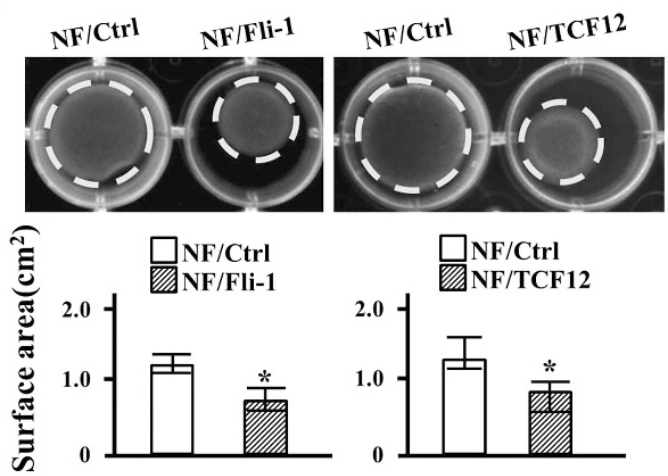

f

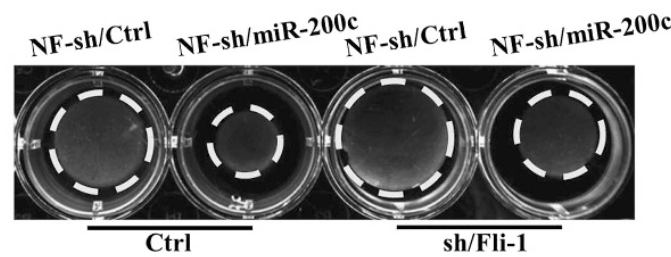

NF-sh/Ctrl NF-sh/miR-141 NF-sh/Ctrl NF-sh/miR-141
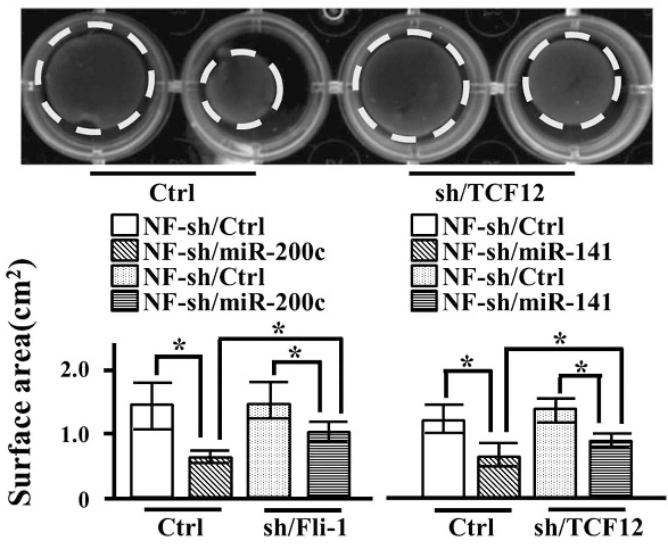
miR-200 s (Figure 5b). The suppression of Fli-1 or TCF12 in CAFs was sufficient to counteract CAF-induced contractile activity (Figure 5c), and overexpressing Fli-1 or TCF12 in NFs endowed these NFs with stronger contractile activity in a 3D collagen I (Col-I) matrix (Figure $5 \mathrm{~d}$ ). Moreover, Fli-1 or TCF12 independently restored the contractile activity of CAF/miR200c and CAF/miR-141 cells (Figure 5e), and shRNA targeted against Fli-1 and TCF12 attenuated Col-I gel contraction by NF-sh/miR-200c or NF-sh/miR-141 cells (Figure 5f). Together, these data provide evidence that miR-200 s and their targets Fli-1 and TCF12 contribute to ECM remodeling by CAFs.

Some ECM remodeling-associated genes are regulated by miR-200 s. As expected, a cohort of ECM-associated genes was identified in CAFs (Figure 6a); this cohort included FN, LOX and MMP, which are potential targets of miR-200 s (Supplementary Table s2). FN and LOX were significantly increased in CAFs (Figure 6b). Further assessments of FN and LOX expression in the engineered fibroblasts indicated that FN could be modulated by the two functional clusters at the mRNA (Supplementary Figures S12a and b) and protein (Figures 6c and d) levels. In addition, FN expression was dependent on TCF12 but not on Fli-1 (Figure 6e and f). LOX was a direct target of miR-200c (Supplementary Figures S12c and d and Figures $6 \mathrm{c}$ and d) and has previously been shown to be a direct target of miR-200b, ${ }^{23}$ and LOX expression in CAFs was regulated in a Fli-1-dependent manner (Figures $6 \mathrm{e}$ and f). In CAFs, the induction of LOX and FN expression by miR-200c and miR-141 was effectively rescued by Fli-1 and TCF12; in contrast, in miR-200c- or miR-141-knockdown NFs, the expression of LOX and FN was compromised by the silencing of Fli-1 and TCF12, respectively (Figures $6 \mathrm{~g}$ and $\mathrm{h}$ ). Taken together, these data demonstrate that FN and LOX are major dysregulated ECM proteins in CAFs and are involved in ECM remodeling induced by miR-200 s and their targets. In addition, FN is a new indirect target of miR-141, and LOX is a new target of miR-200c.

miR-200s and their targets Fli-1 and TCF12 stimulate breast tumor cell invasion and metastasis. miR-200 $\mathrm{s}$ and their targets Fli-1 and TCF12 promote CAF activation and are involved in ECM remodeling, which led us to investigate whether they can affect the topographical organization of the ECM and tumor cell invasion. To address this question, MDAMB-231 cell invasion in a Col-I gel was measured. Restoring miR-200 s in CAFs resulted in thinner and less organized fibroblast-derived collagen fibers, as detected by picrosirius red (PR) staining (Figure 7a), and slowed breast cancer cell invasion in the Col-I gel (Figure 7d). However, examination of the architectural patterns in the Col-I gel embedded with
NF-sh/200 s fibroblasts revealed a thicker and more organized collagen-rich matrix (Figure $7 \mathrm{~b}$ ), and greater MDAMB-231 cell invasion was observed (Figure 7e). Indeed, the loss of Fli-1 or TCF12 in CAFs impeded CAF-induced architecture organization (Figure 7c, upper panel), and MDA-MB-231 invasion in the Col-I gel was subsequently reduced (Figure $7 f$, upper panel). In contrast, the ectopic expression of Fli-1 or TCF12 in NFs produced ECM fibers oriented in parallel patterns, as opposed to the disordered matrices generated by control cells (Figure 7c, bottom panel), with accelerated tumor cell invasion in the Col-I gel (Figure 7f, bottom panel). Furthermore, the re-expression of Fli-1 in CAF/miR-200c cells or TCF12 in CAF/miR-141 cells restored MDA-MB-231 cell invasion in the Col-I gel (Figure $7 \mathrm{~g}$ ). Decreased expression of Fli-1 and TCF12 in NF-sh/miR-200c and NF-sh/miR-141 cells partially counteracted the invasive ability of MDA-MB-231 cells in the Col-I gel (Figure $7 \mathrm{~h}$ ). In addition, in orthotopic mouse models, the tumors generated from MDA-MB-231 cells in combination with CAFs expressing miR-200s and their targets grew slower than those generated from MDA-MB-231 cells mixed with CAF/sh-Ctrl cells (Figure $8 \mathrm{a}$ and Supplementary Figures S13a and c). $\mathrm{ECM}$ deposition in the presence of CAF/miR-200 s, CAF/shFli-1 or CAF/sh-TCF12 cells, as assessed by PR staining, was less aligned than that observed in the presence of CAF/sh-Ctrl cells (Figure 8b). Lungs were harvested 6 weeks after the cell injections; in CAFs, miR-200 s and their targets Fli-1 and TCF12 significantly decreased surface lung metastases and the lung metastatic burden (Figures $8 \mathrm{c}$ and $\mathrm{g}$ ). Collectively, these data support the hypothesis that low levels of miR-200 s in CAFs increase Fli-1 and TCF12 expression and promote stromal ECM remodeling to fuel tumor cell invasion and metastasis.

\section{Discussion}

The microenvironment and tumor cells interact with each other. ${ }^{24}$ The presence of CAFs, a major component of the tumor stroma, signifies tumor progression; however, the complex nature of CAF activation in vivo and the underlying cancer-promoting effects have yet to be explored. Here, we demonstrated that the miR-200 family, including miR-200a, miR-200b, miR-200c and miR-141, were often downregulated in CAFs compared with paired NFs derived from breast cancer patients as well as in NFs co-cultured with breast cancer cells. The downregulation of miR-200s could induce CAF-like characteristics in NFs in terms of increased expression of the CAF markers $a$-SMA and FAP and enhanced migration and invasion activity. Importantly, the contractile activity of CAFs in a Col-I gel was regulated by miR-200 s, suggesting that miR-200 s affect ECM remodeling.

Figure 5 Activated CAFs generated in response to miR-200 s and their targets promote ECM remodeling. Fibroblasts were grown in a Col-I gel and cultured for 2 days. The ability of the indicated fibroblasts to elicit ECM remodeling was evaluated by contraction assays in Col-I gels. Representative images of Col-I gels are shown. The experiments were repeated at least three times, and the data are presented as the mean \pm S.D. ( $n>3 ;{ }^{*} P<0.05$, Student's t-test). (a) Re-expression of the miR-200 s in CAFs reduced ECM remodeling. (b) Knockdown of miR-200 s in NFs enhanced the contractile activity of the indicated fibroblasts in a Col-I gel. (c) Decreasing Fli-1 or TCF12 expression using shRNA in CAFs reduced ECM remodeling. (d) Ectopic expression of Fli-1 or TCF12 in NFs increased ECM remodeling. (e) Restoring the expression of Fli-1 in CAF/miR-200c cells or TCF12 in CAF/miR-141 cells rescued the ECM remodeling of the indicated CAFs. (f) Silencing Fli-1 or TCF12 expression again in NF-sh/miR-200c or NF-sh/miR-141 cells attenuated their contractile activity in Col-I gels 
a

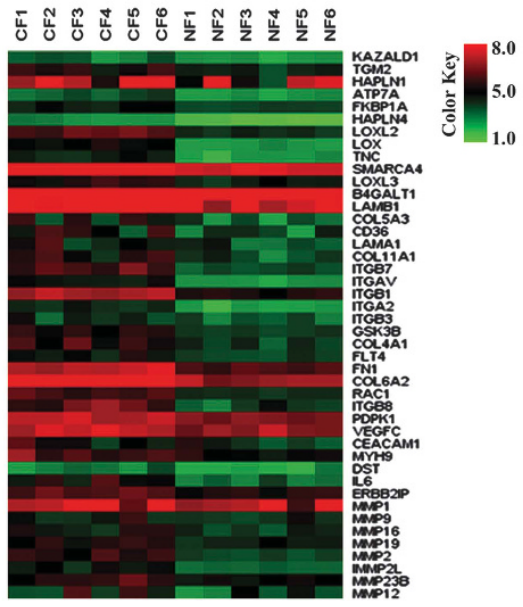

c

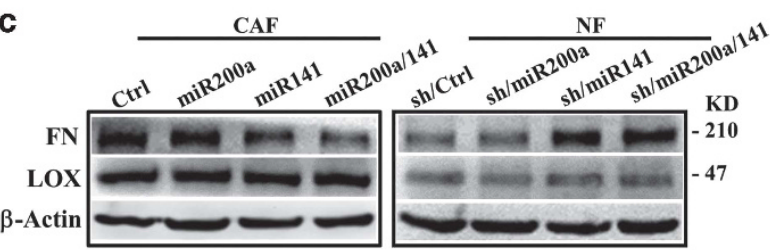

d

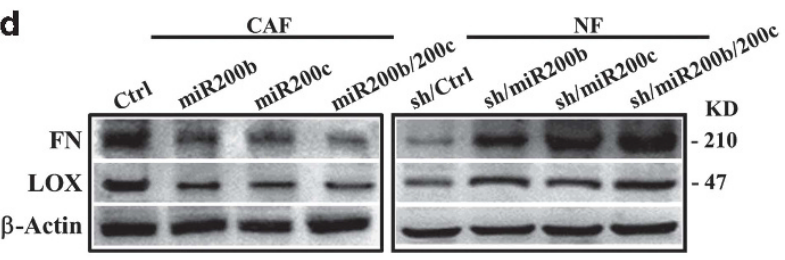

b
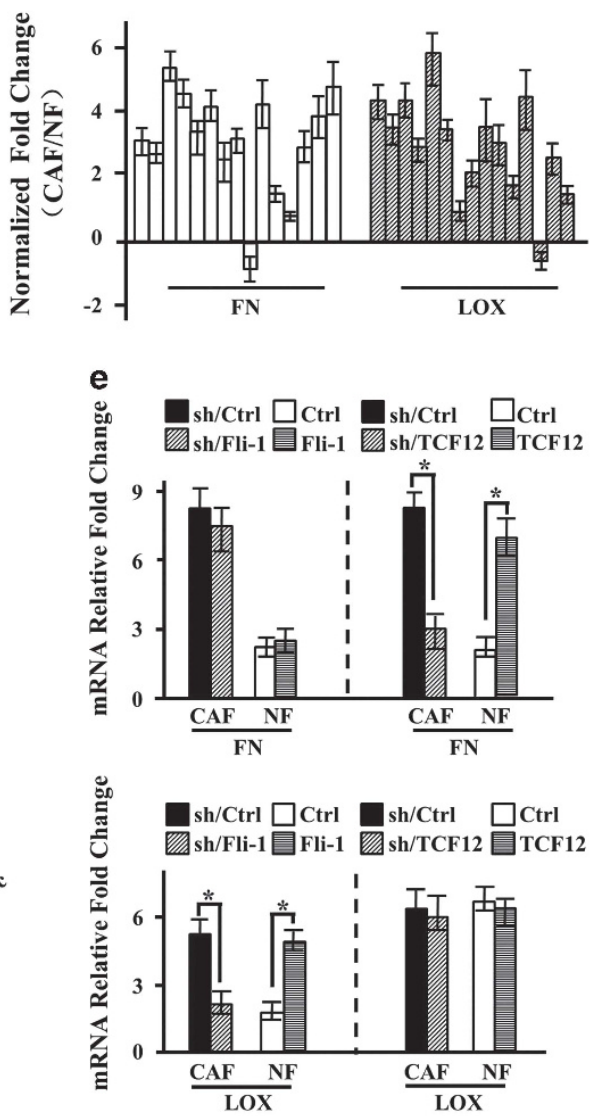

g

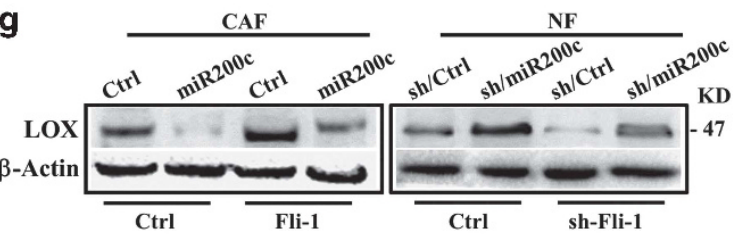

h
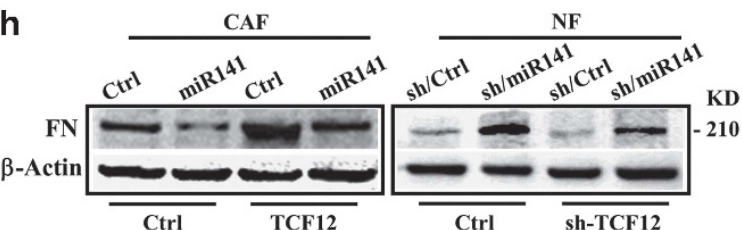

f

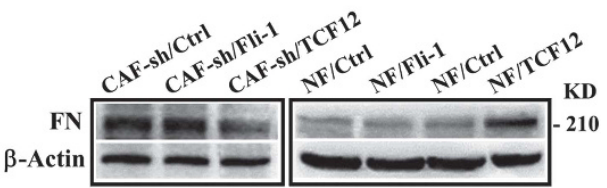

Figure 6 miR-200 s and/or their targets Fli-1 and TCF12 are involved in the expression of ECM-associated genes in CAFs. (a) Heat map illustrating the hierarchical clustering of altered ECM-associated genes in CAFs and paired NFs. (b) qRT-PCR analysis of FN and LOX expression in 15 paired CAF and NF samples that were freshly isolated from breast tumor tissues. U6 was used as an internal control. The data are shown as the normalized fold change in CAFs compared with NFs. (c and d) Western blot analysis of FN and LOX expression in the indicated CAFs or NFs. (e) mRNA expression of FN and LOX as determined by qRT-PCR in the indicated CAFs or NFs. $\beta$-Actin was used as an internal control. The data are presented as the mean \pm S.D. $\left(n=3 ;{ }^{*} P<0.05\right.$, Student's t-test). (f) Western blot analysis of FN and LOX expression in CAF-sh/TCF12 or NF/TCF12 cells. (g) LOX protein expression was analyzed by western blot after re-expressing Fli-1 in CAF/miR-200c cells or knocking down Fli-1 in NF-sh/miR-200c cells. (h) FN protein expression was evaluated by western blot after re-expressing TCF12 in CAF/miR-141 cells or knocking down TCF12 in NF-sh/miR-141 cells

Figure 7 miR-200 s and their targets Fli-1 and TCF12 promote breast cancer cell invasion in ECM. CAFs expressing vectors encoding miR-200 s were grown in a Col-I gel for 10 days and then treated with detergent extraction. MDA-MB-231 breast cancer cells were seeded onto the Col-I gel and cultured for 5 days. The architecture of the paraffinembedded Col-I gel was visualized by PR staining, and the invasive cells in the ECM gel were visualized by H\&E staining; representative images are shown (Scale bars, $100 \mu \mathrm{m}$ ). (a-c) Matrices derived from the indicated fibroblasts were analyzed by PR staining for collagen deposition and orientation. (d-f) MDA-MB-231 cell invasion in the Col-I gel that was remodeled by the indicated fibroblasts. (g) MDA-MB-231 cell invasion in the Col-I gel that was remodeled by the indicated CAFs was determined after ectopically expressing Fli-1 in CAF/miR-200c cells or TCF12 in CAF/miR-141 cells. (h) MDA-MB-231 cell invasion in the Col-I gel that was remodeled by the indicated NFs was investigated after knocking down Fli-1 and miR-200c or TCF12 and miR-141 expression using specific shRNAs 
a

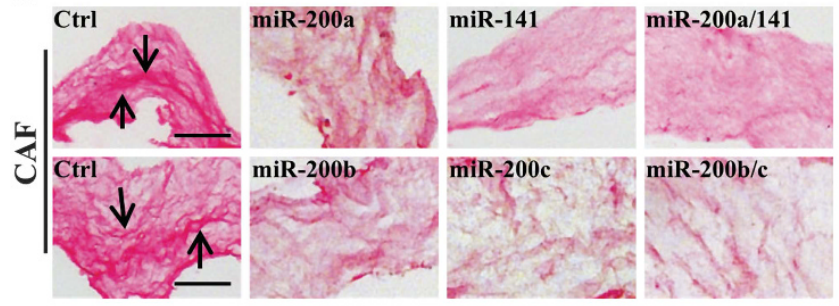

b

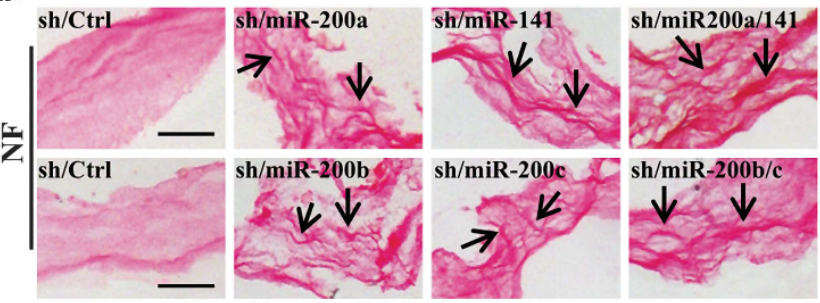

C

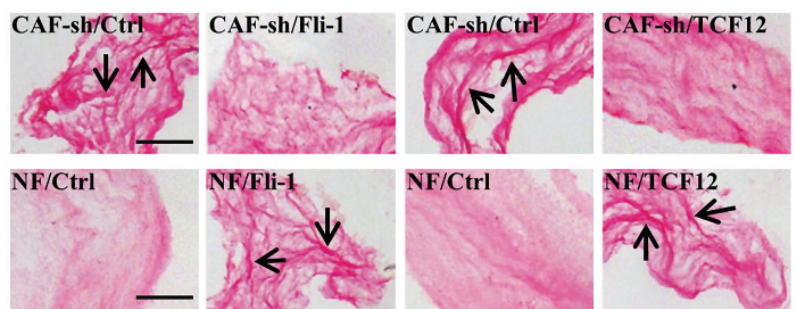

d

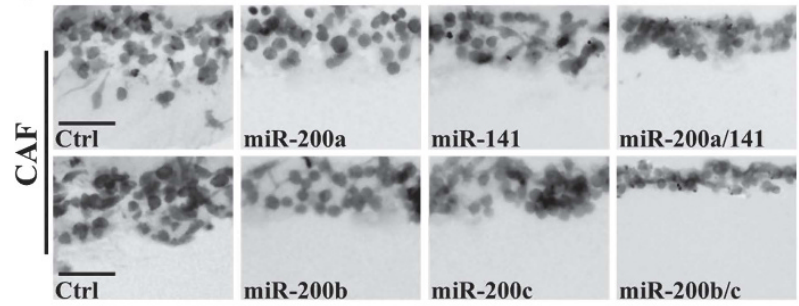

e

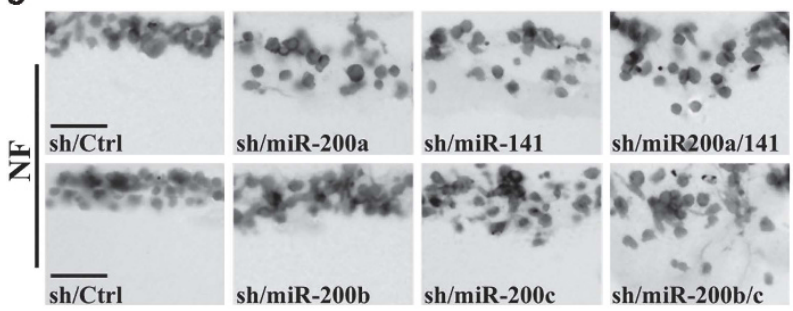

f

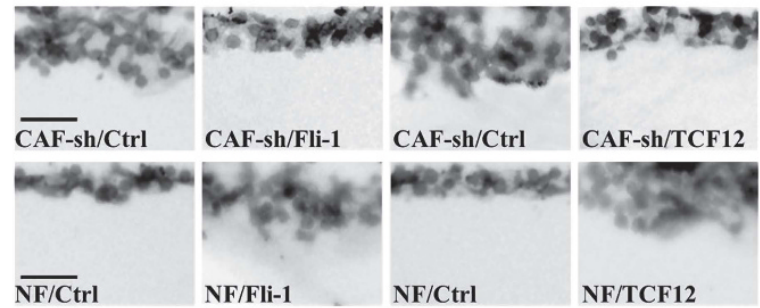

g
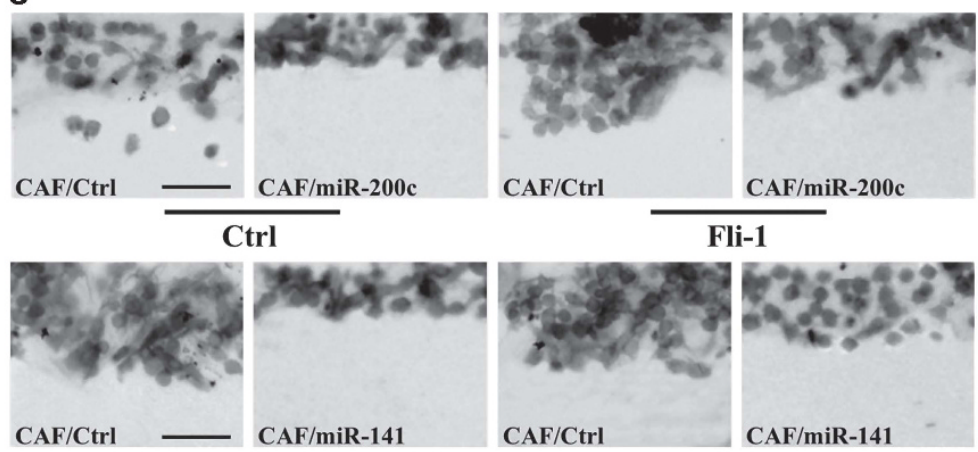

Ctrl

Fli-1

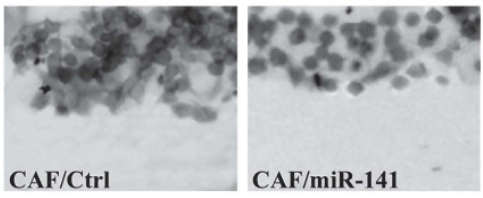

TCF12

h
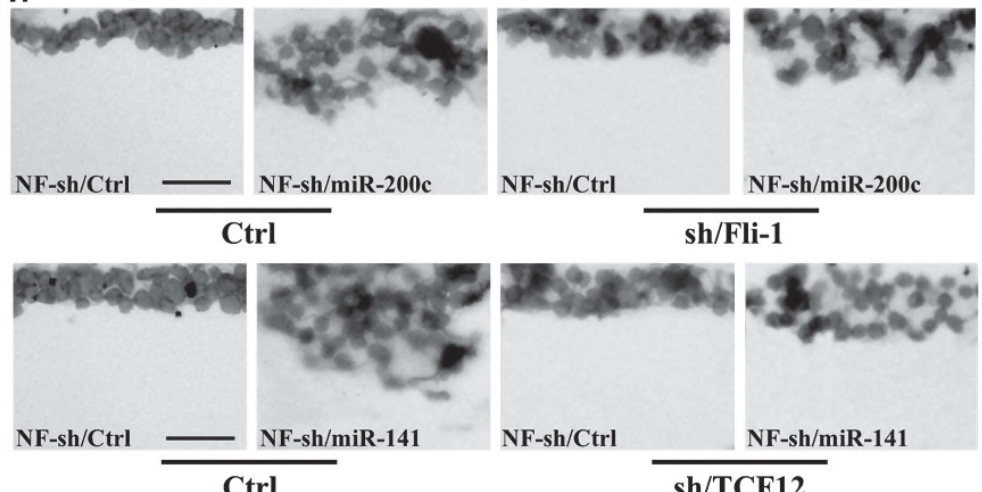

sh/TCF12 
miRNAs control the expression of multiple targets and participate in a wide variety of biological processes. ${ }^{23}$ They have been described as oncogenes or tumor suppressors in different tumor types, wherein they contribute to proliferation, invasion and metastasis. However, the precise role of miRNAs in the tumor stroma has been insufficiently explored. A limited number of miRNAs that are differentially expressed in CAFs have been determined to mediate the cancer-promoting abilities of these cells. For example, the suppression of miR-31 in endometrial CAFs, ${ }^{25}$ the downregulation of miR-15 and miR-16 in prostate CAFs ${ }^{26}$ and the attenuated expression of miR-214 and miR-31 in ovarian $\mathrm{CAFs}^{27}$ have all been shown to promote cancer cell survival, proliferation and migration. In the present work, we determined that miR-200 s were downregulated in breast cancer CAFs, thereby promoting CAF migration and invasion. These data are consistent with previous data showing that miR-26b induces CAF migration and invasion in estrogen receptor-positive breast cancer. $^{28}$ Taken together, the evidence suggests that disordered tumor-inhibiting miRNAs in CAFs are likely key mediators of activated CAF-induced cancer metastasis.

The regulatory mechanisms that control CAF features and activation have been partially revealed. Among the known stimulators of fibroblasts, paracrine factors, such as transforming growth factor- $\beta$ (TGF- $\beta$ ), ${ }^{29}$ CXCL12 (ref 17) and platelet-derived growth factor, ${ }^{30}$ have been implicated as major regulators of CAFs. Transcription factors interact with many co-regulatory partners to facilitate gene-specific responses and drive distinct biological processes. However, their regulatory role in CAF activation has not been generally described. In fact, lung cancer properties can be induced in CAFs by FoxF1 through hedgehog signaling. ${ }^{31}$ The generation of CAFs via the treatment of NFs with conditioned medium from MDA-MB-231 cells results in increased expression of numerous transcription factors associated with proliferation, differentiation and the inflammatory response. ${ }^{32}$ However, these data may obscure the noteworthy concept that the activation of key transcription factors is sufficient to induce CAF properties. Here we first demonstrated that the loss of miR-200s had an important role in reprograming NFs into CAFs and regulating the functions of activated CAFs. Importantly, Fli-1 and TCF12, two key transcriptional molecules, effectively induced the CAF properties that were repressed by miR-200 s; these data provide new insights into CAF activation.

Fli-1 and TCF12 are the candidate effectors of miR-200 s in breast cancer CAFs. The miR-200c-mediated suppression of Fli-1 and the miR-141-mediated inhibition of TCF12 partially explain the effects of the miR-200 family on the traits and contractility of activated CAFs. The levels of Fli-1 and TCF12 are closely correlated with malignant transformation and cellular differentiation. Fli-1 is critical for embryogenesis and hematopoiesis, ${ }^{33,34}$ and TCF12 has been demonstrated to function as a mediator of the development of $\mathrm{T}$ cells, ${ }^{35}$ lymphocytes, ${ }^{36}$ skeletal muscle ${ }^{37}$ and oligodendrocytes. ${ }^{38}$ The conversion of NFs into CAFs is actually a process of cell redifferentiation. The roles of Fli-1 and TCF12 in the upregulation of CAF markers and the increases in migratory and invasive behavior highlight the influence of stromal miRNA-induced transcription factors on CAF activation and cancer progression.
The stiffness of the ECM, which is dependent on the composition of the microenvironment as well as the organization and complexity of the ECM itself, perturbs the tissue architecture and enhances tumor growth and invasion. ${ }^{39,40}$ Because of their abnormal contractile activity and deposition of ECM components, stromal CAFs can remodel the ECM and thus act as critical modulators of the metastatic potential of tumor cells. ${ }^{2,40}$ In the present work, we determined the effects of miR-200 s and their target genes Fli-1 and TCF12 on the contractile activity of CAFs and on the invasion and metastasis of breast cancer cells in ECM deposited by engineered fibroblasts in vitro and in vivo. We found that miR-200c directly or indirectly regulated LOX expression through its target Fli- 1 and that miR-141 induced FN expression through TCF12. Hence, miR-200 s and their targets play a key role in ECM remodeling.

The expression levels of LOX and FN were found to be the most discrepant between paired CAFs and NFs in breast cancer, and these proteins had a pivotal influence on ECM remodeling. LOX is an enzyme necessary for Col-I crosslinking that increases fibrillar collagen deposition and linearization; ${ }^{41}$ it has been shown to contribute to the establishment and maintenance of the pre-metastatic niche ${ }^{42}$ and to be a hallmark of lymph node metastasis in several squamous carcinomas. ${ }^{43} \mathrm{FN}$ binds to collagens and affects the organization of collagen fibers, thereby assembling an intricate fibrillar network that transmits biochemical and mechanical stimuli from the matrix to the cytoskeleton through integrins. ${ }^{44}$ These reports support our results that miR-200 s and their targets Fli1 and TCF12 influenced fibroblast characteristics and that the deposited ECM-associated proteins LOX and FN facilitated the malignant breast cancer cell invasion. To the best of our knowledge, this is the first report to demonstrate that the appearance of the ECM was modulated by miR-200 s and their directly regulated transcription factors Fli-1 and TCF12.

Collectively, the present study provides data that have significant implications regarding our understanding of how miR-200 s influence CAF activation and ECM remodeling, which trigger tumor cell invasion and metastasis. The ability of miR-200 s to impede the conversion of NFs to CAFs may be clinically useful.

\section{Materials and Methods}

Tissue samples. Human breast tumor tissues and adjacent normal mammary tissues (at least $5 \mathrm{~cm}$ away from the tumor) were obtained from patients with breast cancer without previous radiotherapy or neoadjuvant chemotherapy at the First Affiliated Hospital of Chongqing Medical University. The study was approved by the Ethics Committee of Chongqing Medical University.

Isolation of primary fibroblasts and cell culture. Fibroblasts were isolated as described previously. ${ }^{45}$ In brief, tumor tissue and corresponding normal mammary tissues were minced and digested in collagenase type I (C0130, Sigma, St Louis, MO, USA). The mixtures were then cultured with DMEM (Invitrogen, Carlsbad, CA, USA) containing 10\% FBS (GIBCO-BRL) in a humidified atmosphere containing $5 \% \mathrm{CO}_{2}$ at $37^{\circ} \mathrm{C}$ until the fibroblasts attached to the plastic. All the primary fibroblasts used in the experiments were at passages 3 to 6 .

Immortalized CAFs and NFs (immortalized using pBABE-hygro-hTERT), which have been described previously, ${ }^{45}$ and GES cells were maintained in DMEM with 10\% FBS. MDA-MB-231, MCF-7, MDA-MB-453, MDA-MB-468 and SGC-7901 cells were cultured in RPMI 1640 medium (Invitrogen) with 10\% FBS, and BT474 cells were cultured in RPMl 1640 medium with 10\% FBS and insulin. MCF10A cells were cultured in DMEM/F12 medium supplemented with $5 \%$ horse serum, $10 \mathrm{mg} / \mathrm{ml}$ insulin, $0.5 \mathrm{mg} / \mathrm{ml}$ hydrocortisone, $20 \mathrm{ng} / \mathrm{ml}$ recombinant epidermal growth factor and $100 \mathrm{ng} / \mathrm{ml}$ cholera toxin. 
a

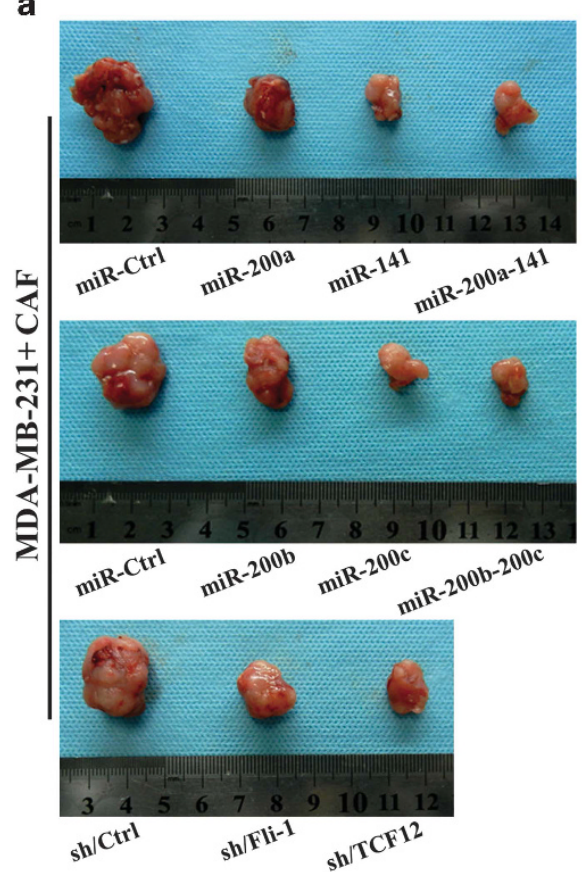

e

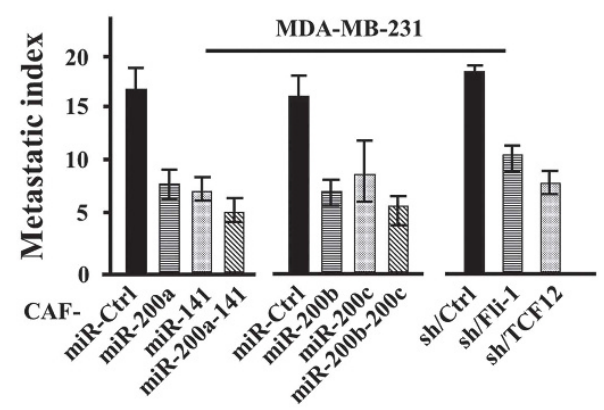

f

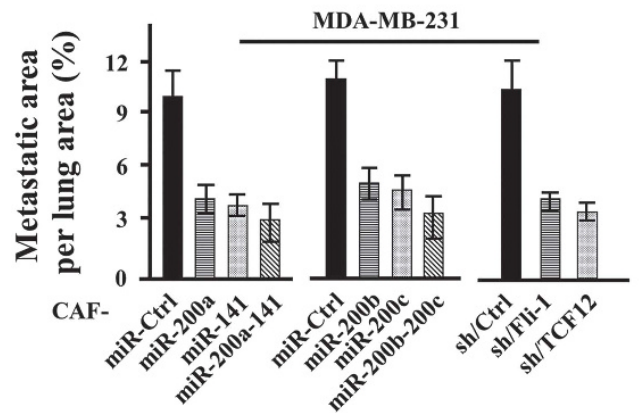

b
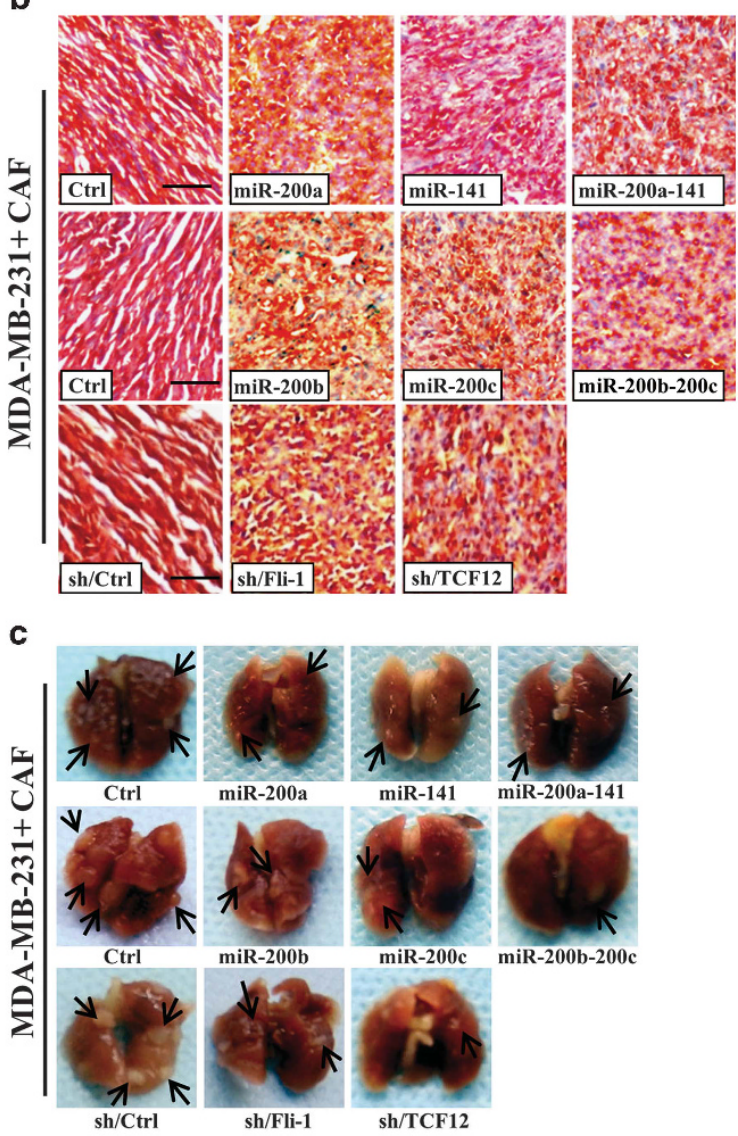

d
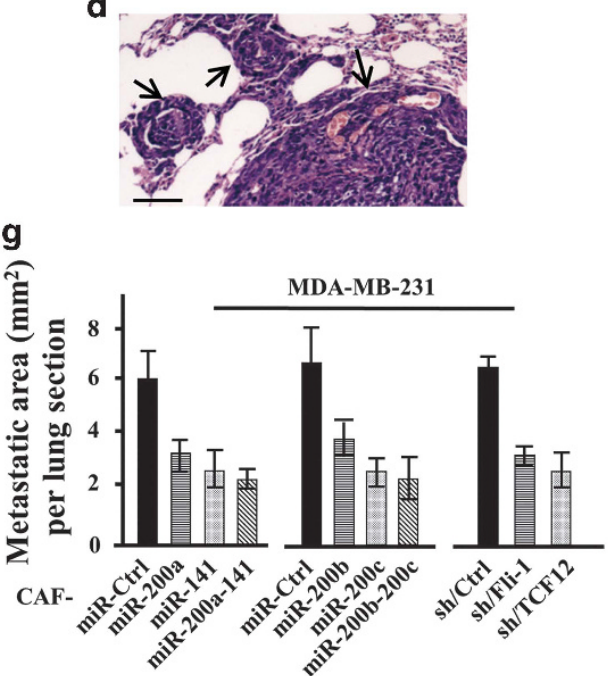

Figure 8 miR-200 s and their targets Fli-1 and TCF12 suppress the metastatic potential of malignant cells in vivo. MDA-MB-231 breast cancer cells were subcutaneously injected into nude mice in combination with CAF/miR-200 s, CAF/sh-Fli-1, CAF/sh-TCF12 or control cells; 45 days following injection, the mice were killed. Representative images (a) indicating tumor size in mice injected with MDA-MB-231 cells mixed with the indicated CAFs and (b) illustrating PR-stained tumor samples. Red, collagen fibers; Blue, nuclei. Scale bars, $100 \mu \mathrm{m}$. Representative images of (c) a whole lung fixed in formalin for determining surface metastases and (d) an H\&E-stained lung section confirming the presence of pulmonary metastases. The arrows indicate metastatic nodules. Graphs of the fold change in (e) the number of metastatic foci per lung, ( $\mathrm{f}$ ) the percentage of metastatic area to total lung area and $(\mathbf{g})$ the area per lung section in five random sections per animal 
For co-culture experiments, cancer cells or normal epithelial cells and NFs were used at a ratio of 3:1 by seeding NFs or cancer cells in six-well plates and then seeding cancer cells or NFs in the upper chambers of 4- $\mu \mathrm{m}$-pore Boyden chambers (Millipore, Darmstadt, Germany). Total RNA and protein were extracted at 15 and 30 days. NFs were cultured with conditioned media from breast cancer cells or normal epithelial cells, the supernatant was changed daily, and total RNA was extracted after 21 days.

Plasmid construction, inhibitors and mimics. pLenti4.1-puro-pri-miR200b-200a-429/pri-miR-200c-141 was kindly donated by Dr Gregory J Goodall (The University of Adelaide, Adelaide, Australia), and pcDNA3-Fli-1 was a gift from Dr Yoshiaki Okada (Osaka University, Osaka, Japan). pLenti4.1-puro-pre-miR-200a/ 200b/200c/141 and pLenti4.1-puro-Fli-1 were constructed by PCR subcloning into the pLenti4.1-puro vector. GV-puro-TCF12 was purchased from GeneChem (Shanghai, China). All the synthetic shRNA oligonucleotides (Invitrogen) specifically targeting the miR-200a/200b/200c/141, Fli-1 or TCF12 genes were inserted into the pLVX-shRNA1 lentivector (Clontech, Palo Alto, CA, USA). To generate the luciferase reporters used to assay the direct targeting of Fli- 1 and TCF12 by miR-200 s, the synthetic oligonucleotides (Invitrogen) corresponding to the wild-type and mutated binding sites of miR-200 s in the 3'-UTR of Fli-1 or TCF12 were cloned into the pMIR-Report vector (Ambion, Austin, TX, USA) at the Spe 1 and Hind III restriction sites. The binding sites were identified using TargetScan (MIT, www.targetscan.org). Mimics and inhibitors of miR-200 s and the corresponding controls were purchased from GenePharma (Shanghai, China). The sequences described above are provided in Supplementary Tables S3-S5.

RNA preparation and qRT-PCR. Total RNA was isolated using Trizol (15596-026, Invitrogen) according to the manufacturer's instructions. The purified RNAs were subjected to reverse transcription using a PrimeScript RT Reagent Kit (RR047A, TaKaRa, Dalian, China). qRT-PCR was performed with SYBR Premix Ex Taq II (RR820A, TaKaRa) according to the manufacturer's instructions. Triplicate CDNA samples were assayed, and the average threshold cycle $(C t)$ values were calculated. Relative gene expression was measured as $2^{\mathrm{Ct}(\text { internal control) - Ct(gene) }}$. The utilized primers are listed in Supplementary Tables S6-S7. All the experiments were performed at least three times.

Western blot analysis. Western blot analysis were performed as described previously. ${ }^{46}$ In brief, proteins in the cell lysates were separated by $10 \%$ SDS-PAGE and subjected to western blotting. The following antibodies were used: Fli-1 (Sc-356, rabbit polyclonal antibody, 1:200, Santa Cruz, Santa Cruz, TX, USA), TCF12 (Sc-357, rabbit polyclonal antibody, $1: 200$, Santa Cruz), $\alpha$-SMA (ab5694, rabbit polyclonal antibody, 1:1000, Abcam, New Territories, Hong Kong), FAP (ab53066, rabbit polyclonal antibody, 1:1000, Abcam), FN (BS1644, rabbit polyclonal antibody, 1:500, BioWorld, Nanjing, China), LOX (AB60069a, rabbit polyclonal antibody, 1:500, Shenggong, Shanghai, China), and $\beta$-actin $(1: 1000$, Boster, Wuhan, China). The appropriate horseradish peroxidase-conjugated secondary antibodies were subsequently applied, and immunodetection was conducted using an enhanced chemiluminescence system (Amersham Pharmacia Biotech, Freiburg, Germany). Images were captured using Scion image software.

Enzyme-linked immunosorbent assay. Conditioned media from 30-day co-cultures or from $3 \times 10^{5}$ breast cancer cells (MCF-10 A, BT474, MDA-MB-453, MCF-7 or MDA-MB-468) were harvested, and the concentrations of IL- 6 and TGF $\beta 1$ were measured by ELISA (ExCell Bio, Shanghai, China) according to the manufacturer's instructions. The absorbance $(450 \mathrm{~nm})$ of each sample was analyzed using a standard ELISA plate reader.

Luciferase reporter assay. A total of $1 \times 10^{5}$ HEK-293 cells or CAFs were seeded in 24-well plates and co-transfected with a total of $800 \mathrm{ng}$ of expression plasmids containing miR-200 s or pMIR-Fli-1/TCF12 (wild-type or mutant) and the control plasmid pRL-TK (Promega, Madison, WI, USA) using Lipofectamine 2000 (Invitrogen). After culture for $48 \mathrm{~h}$, the lysates were collected, and Renilla and firefly luciferase activities were measured with a Dual-Luciferase Reporter System (E1910, Promega).

Wound-healing and invasion assays. Fibroblasts were seeded in six-well plates. Confluent cell monolayers were scratched with a sterile pipette tip, and the medium was replaced with serum-free DMEM. The mobility of the fibroblasts was monitored by phase contrast microscopy (Nikon TE2000-U, Japan) at the designated time point, and ImageJ software was utilized to measure the wounded area as previously described. ${ }^{45}$
Fibroblast invasion was measured using the transwell assay as described previously. ${ }^{45}$ In brief, $2.5 \times 10^{4}$ CAFs or NFs in $200 \mathrm{ml}$ of serum-free DMEM were seeded in the top well of Boyden chambers coated with Matrigel (356231, Corning BioCoat, Bedford, USA). Images were captured using a Nikon Eclipse 80i microscope (Eclipse 80i, Tokyo, Japan).

For the breast cancer cell invasion assay in ECM, $5 \times 10^{4}$ fibroblasts were embedded in a Col-I gel (354249, Corning BioCoat) at a final concentration of $1 \mathrm{mg} /$ $\mathrm{ml}$. After 10 days, the gels were incubated in extraction buffer ( $0.5 \%$ Triton-X-100 and $20 \mathrm{mM} \mathrm{NH}_{4} \mathrm{OH}$ in PBS) to kill the fibroblasts and then washed several times with complete media ( $>30$ min per wash). A total of $3 \times 10^{5}$ breast cells were then plated on top of the ECM. After 5 days of incubation, the cultures were fixed using $4 \%$ paraformaldehyde plus $0.25 \%$ glutaraldehyde in PBS and embedded in paraffin; then, lengthwise sections were processed for hematoxylin and eosin (H\&E) staining. Images were captured using a Nikon Eclipse 80i microscope (Eclipse 80i, Tokyo, Japan) and subsequently collected, imported and incorporated into electronic figures using NIS-Elements software, ImageJ and Microsoft PowerPoint 2010.

Collagen-remodeling assay. A total of $2 \times 10^{5}$ fibroblasts were embedded in a rat tail Col-I gel as described above. The mixture $(500 \mu \mathrm{l})$ was dispensed into single wells of a 24 -well plate and incubated at $37^{\circ} \mathrm{C}$ for $30 \mathrm{~min}$. After polymerization, the collagen gels were freed from the wells using a 100- $\mu$ l pipette tip; subsequently, complete DMEM was added to the wells. Two days later, the diameters of the collagen gels were measured. Images were captured using Gel Doc XR (Bio-Rad, Segrate, Italy) and analyzed using Quantity One software. Electronic figures were imported and incorporated using ImageJ and Microsoft PowerPoint 2010.

IHC, immunofluorescence (IF) and PR staining. IHC analysis was performed as described previously. ${ }^{47}$ In brief, the sections were incubated with a primary rabbit anti-TCF12 or Fli-1 polyclonal antibody (1:100 or 1:50, Santa Cruz) overnight at $4{ }^{\circ} \mathrm{C}$ and for $1 \mathrm{~h}$ at room temperature. The pathology scoring of the tissues was performed as described previously. Nuclear expression of Fli-1 and TCF12 was graded from 0 to $3+$ (0, no staining; $1+, 1 \%-25 \% ; 2+, 26 \%-50 \% ; 3+$, $>50 \%$ nuclear staining). The staining intensities of TCF12/Fli-1 were assessed by examining $80 \%$ of the cell population. ${ }^{46}$ Images were captured using a Nikon Eclipse 80i microscope (Eclipse 80i).

For IF, CAFs and NFs were grown on prepared coverslips to $80 \%$ confluence and then fixed within $4 \%$ paraformaldehyde. After treatment with $0.1 \%$ Triton-X-100 and incubation with a $5 \%$ goat serum solution, the cells were incubated with specific antibodies against TCF12/Fli-1 (1: 100; Santa Cruz), $\alpha$-SMA (1:200; Abcam), FAP (1:200; Abcam) or FITC-Phalloidin (1: 40; Sigma); normal rabbit lgG was used as a negative control. The sections were then treated with a FITC-labeled goat anti-rabbit secondary antibody $(1: 200$, Sigma) and mounted in aqueous medium containing DAPI (Vector Lab, Beijing, China). IF images were captured using a Nikon Eclipse 80i microscope (Eclipse 80i).

Collagen deposition and organization in paraffin-embedded specimens and Col-I gels from the co-culture system were visualized by standard PR staining with Picric acid/Sirius red (DC0040, Lengene, Beijing, China). Images were captured using a Nikon Eclipse 80i microscope (Eclipse 80i), analyzed using NIS-Elements software and imported and incorporated into electronic figures using ImageJ and Microsoft PowerPoint 2010.

Orthotopic xenografts and lung metastasis analysis. The animal experiments were approved by the animal use committees at Chongqing Medical University. The mice were housed in the Animal Care Facility at Chongqing Medical University. A total of $2 \times 10^{6}$ MDA-MB-231 cells mixed with $2 \times 10^{6} \mathrm{CAF} / \mathrm{Ctrl}$ or engineered CAF cells in $200 \mu \mathrm{l}$ of PBS: Matrigel at a 1:1 ratio were subcutaneously injected into 4-week-old female nude mice obtained from HFK BIOSCIENCE (Beijing, China). Primary tumors were palpable 7 to 12 days after implantation. Tumor growth was determined by measuring the two axes of the tumor every four days. The lungs were serially sectioned into $5-\mu \mathrm{m}$-thick tissue sections and stained with H\&E for a subsequent blinded evaluation. ${ }^{47,48}$ On each slide, metastases were counted, and the metastatic and total lung section areas were measured using ImageJ software.

Statistical analysis. Statistical analysis were performed using SPSS Inc., (Chicago, IL, USA) standard version 13.0. The data are presented as the mean \pm S.D. from at least three independent experiments. ANOVA followed by the Student-Newman-Keuls multiple comparisons test was used for comparisons between multiple groups, and Student's t-test was used for single comparisons between two groups. Values of $P<0.05$ were considered significant. 


\section{Conflict of Interest}

The authors declare no conflict of interest.

Acknowledgements. This work was supported in part by the National Natural Science Foundation of China (NSFC 81402180, NSFC 81472476 and NSFC 81072147), the Doctoral Fund of the Ministry of Education, China (20125503110001) and the Scientific Research Foundation for the Returned Overseas Chinese Scholars, Ministry of Education, China ([2011]508).

1. Paszek MJ, Weaver VM. The tension mounts: mechanics meets morphogenesis and malignancy. J Mammary Gland Biol Neoplasia 2004; 9: 325-342.

2. Paszek MJ, Zahir N, Johnson KR, Lakins JN, Rozenberg Gl, Gefen A et al. Tensional homeostasis and the malignant phenotype. Cancer Cell 2005; 8: 241-254.

3. Zaman MH, Trapani LM, Sieminski AL, Mackellar D, Gong H, Kamm RD et al. Migration of tumor cells in 3D matrices is governed by matrix stiffness along with cell-matrix adhesion and proteolysis. Proc Natl Acad Sci USA 2006; 103: 10889-10894.

4. Goetz JG, Minguet S, Navarro-Lerida I, Lazcano JJ, Samaniego R, Calvo E et al. Biomechanical remodeling of the microenvironment by stromal caveolin-1 favors tumor invasion and metastasis. Cell 2011; 146: 148-163.

5. Calvo F, Ege N, Grande-Garcia A, Hooper S, Jenkins RP, Chaudhry SI et al. Mechanotransduction and YAP-dependent matrix remodelling is required for the generation and maintenance of cancer-associated fibroblasts. Nat Cell Biol 2013; 15: 637-646.

6. Zhao L, Sun Y, Hou Y, Peng Q, Wang L, Luo $\mathrm{H}$ et al. MiRNA expression analysis of cancerassociated fibroblasts and normal fibroblasts in breast cancer. Int J Biochem Cell Biol 2012; 44: 2051-2059.

7. Oba S, Kumano S, Suzuki E, Nishimatsu H, Takahashi M, Takamori H et al. miR-200b precursor can ameliorate renal tubulointerstitial fibrosis. PLoS One 2010; 5: e13614.

8. Chen Y, Ge W, Xu L, Qu C, Zhu M, Zhang W et al. miR-200b is involved in intestinal fibrosis of Crohn's disease. Int J Mol Med 2012; 29: 601-606.

9. Burk U, Schubert J, Wellner U, Schmalhofer O, Vincan E, Spaderna S et al. A reciprocal repression between ZEB1 and members of the miR-200 family promotes EMT and invasion in cancer cells. EMBO Rep 2008; 9: 582-589.

10. Saydam O, Shen Y, Wurdinger T, Senol O, Boke E, James MF et al. Downregulated microRNA-200a in meningiomas promotes tumor growth by reducing E-cadherin and activating the Wnt/beta-catenin signaling pathway. Mol Cell Biol 2009; 29: 5923-5940.

11. Korpal M, Lee ES, Hu G, Kang Y. The miR-200 family inhibits epithelial-mesenchymal transition and cancer cell migration by direct targeting of $\mathrm{E}$-cadherin transcriptional repressors ZEB1 and ZEB2. J Biol Chem 2008; 283: 14910-14914.

12. Howe EN, Cochrane DR, Richer JK. Targets of miR-200c mediate suppression of cell motility and anoikis resistance. Breast Cancer Res 2011; 13: R45.

13. Chen J, Tian W, Cai H, He H, Deng Y. Down-regulation of microRNA-200c is associated with drug resistance in human breast cancer. Med Oncol 2012; 29: 2527-2534.

14. Aydogdu E, Katchy A, Tsouko E, Lin CY, Haldosen LA, Helguero L et al. MicroRNAregulated gene networks during mammary cell differentiation are associated with breast cancer. Carcinogenesis 2012; 33: 1502-1511.

15. Shimono Y, Zabala M, Cho RW, Lobo N, Dalerba P, Qian D et al. Downregulation of miRNA-200c links breast cancer stem cells with normal stem cells. Cell 2009; 138: 592-603.

16. Lin CH, Jackson AL, Guo J, Linsley PS, Eisenman RN. Myc-regulated microRNAs attenuate embryonic stem cell differentiation. EMBO J 2009; 28: 3157-3170.

17. Kojima Y, Acar A, Eaton EN, Mellody KT, Scheel C, Ben-Porath I et al. Autocrine TGF-beta and stromal cell-derived factor-1 (SDF-1) signaling drives the evolution of tumor-promoting mammary stromal myofibroblasts. Proc Natl Acad Sci USA 2010; 107: 20009-20014.

18. Rokavec M, Wu W, Luo JL. IL6-mediated suppression of miR-200c directs constitutive activation of inflammatory signaling circuit driving transformation and tumorigenesis. Mol Cell 2012; 45: 777-789

19. Gregory PA, Bracken CP, Smith E, Bert AG, Wright JA, Roslan S et al. An autocrine TGFbeta/ZEB/miR-200 signaling network regulates establishment and maintenance of epithelialmesenchymal transition. Mol Biol Cell 2011; 22: 1686-1698.

20. Peng Q, Zhao L, Hou Y, Sun Y, Wang L, Luo H et al. Biological characteristics and genetic heterogeneity between carcinoma-associated fibroblasts and their paired normal fibroblasts in human breast cancer. PLoS One 2013; 8: e60321.

21. Gyorffy B, Lanczky A, Eklund AC, Denkert C, Budczies J, Li Q et al. An online survival analysis tool to rapidly assess the effect of 22,277 genes on breast cancer prognosis using microarray data of 1,809 patients. Breast Cancer Res Treat 2010; 123: 725-731.

22. Kalluri R, Zeisberg M. Fibroblasts in cancer. Nat Rev Cancer 2006; 6: 392-401.

23. Ambros V. The functions of animal microRNAs. Nature 2004; 431: 350-355.

24. Lu P, Weaver VM, Werb Z. The extracellular matrix: a dynamic niche in cancer progression. J Cell Biol 2012; 196: 395-406

25. Aprelikova O, YuX, Palla J, Wei BR, John S, Yi M et al. The role of miR-31 and its target gene SATB2 in cancer-associated fibroblasts. Cell Cycle 2010; 9: 4387-4398.
26. Musumeci M, Coppola V, Addario A, Patrizii M, Maugeri-Sacca M, Memeo L et al. Control of tumor and microenvironment cross-talk by miR-15a and miR-16 in prostate cancer. Oncogene 2011; 30: 4231-4242.

27. Mitra AK, Zillhardt M, Hua Y, Tiwari P, Murmann AE, Peter ME et al. MicroRNAs reprogram normal fibroblasts into cancer-associated fibroblasts in ovarian cancer. Cancer Discov 2012; 2: 1100-1108.

28. Zhang $Y$, Yang Z, Whitby $R$, Wang $L$. Regulation of miR-200c by nuclear receptors PPARalpha, LRH-1 and SHP. Biochem Biophys Res Commun 2011; 416: 135-139.

29. Lohr M, Schmidt C, Ringel J, Kluth M, Muller P, Nizze H et al. Transforming growth factorbeta1 induces desmoplasia in an experimental model of human pancreatic carcinoma. Cancer Res 2001; 61: 550-555

30. Rizvi S, Mertens JC, Bronk SF, Hirsova P, Dai H, Roberts LR et al. Platelet-derived growth factor primes cancer-associated fibroblasts for apoptosis. J Biol Chem 2014; 289: 22835-22849.

31. Bao B, Wang Z, Ali S, Ahmad A, Azmi AS, Sarkar SH et al. Metformin inhibits cell proliferation, migration and invasion by attenuating CSC function mediated by deregulating miRNAs in pancreatic cancer cells. Cancer Preven Res 2012; 5: 355-364.

32. Venneti S, Boateng LA, Friedman JR, Baldwin DA, Tobias JW, Judkins AR et al. MiRNA-9 and MiRNA-200a distinguish hemangioblastomas from metastatic clear cell renal cell carcinomas in the CNS. Brain Pathol 2011; 22: 522-529.

33. Brown LA, Rodaway AR, Schilling TF, Jowett T, Ingham PW. Patient RK, et al Insights into early vasculogenesis revealed by expression of the ETS-domain transcription factor Fli-1 in wild-type and mutant zebrafish embryos. Mech Dev 2000; 90: 237-252.

34. Huang $\mathrm{H}$, Yu M, Akie TE, Moran TB, Woo AJ, Tu N et al. Differentiation-dependent interactions between RUNX-1 and FLI-1 during megakaryocyte development. Mol Cell Biol 2009; 29: 4103-4115.

35. Braunstein M, Anderson MK. HEB in the spotlight: transcriptional regulation of T-cell specification, commitment, and developmental plasticity. Clin Dev Immunol 2012; 2012: 678705.

36. Liu YN, Yin JJ, Abou-Kheir W, Hynes PG, Casey OM, Fang L et al. MiR-1 and miR-200 inhibit EMT via Slug-dependent and tumorigenesis via Slug-independent mechanisms. Oncogene 2012; 32: 296-306.

37. Kumar MS, Hendrix JA, Johnson AD, Owens GK. Smooth muscle alpha-actin gene requires two E-boxes for proper expression in vivo and is a target of class I basic helix-loop-helix proteins. Circ Res 2003; 92: 840-847.

38. Sussman CR, Davies JE, Miller RH. Extracellular and intracellular regulation of oligodendrocyte development: roles of Sonic hedgehog and expression of $E$ proteins. Glia 2002; 40: 55-64.

39. Levental KR, Yu H, Kass L, Lakins JN, Egeblad M, Erler JT et al. Matrix crosslinking forces tumor progression by enhancing integrin signaling. Cell 2009; 139: 891-906.

40. Cukierman E, Bassi DE. Physico-mechanical aspects of extracellular matrix influences on tumorigenic behaviors. Semin Cancer Biol 2010; 20: 139-145.

41. Pfeiffer BJ, Franklin CL, Hsieh FH, Bank RA, Phillips CL. Alpha 2(I) collagen deficient oim mice have altered biomechanical integrity, collagen content, and collagen crosslinking of their thoracic aorta. Matrix Biol 2005; 24: 451-458.

42. Erler JT, Bennewith KL, Cox TR, Lang G, Bird D, Koong A et al. Hypoxia-induced lysyl oxidase is a critical mediator of bone marrow cell recruitment to form the premetastatic niche. Cancer Cell 2009; 15: 35-44

43. Albinger-Hegyi A, Stoeckli SJ, Schmid S, Storz M, lotzova G, Probst-Hensch NM et al. Lysyl oxidase expression is an independent marker of prognosis and a predictor of lymph node metastasis in oral and oropharyngeal squamous cell carcinoma (OSCC). Int J Cancer 2010; 126: 2653-2662.

44. Sottile J, Hocking DC. Fibronectin polymerization regulates the composition and stability of extracellular matrix fibrils and cell-matrix adhesions. Mol Biol Cell 2002; 13: 3546-3559.

45. Wang L, Hou Y, Sun Y, Zhao L, Tang X, Hu P et al. c-Ski activates cancer-associated fibroblasts to regulate breast cancer cell invasion. Mol Oncol 2013; 7: 1116-1128.

46. Hu P, Yang J, Hou Y, Zhang H, Zeng Z, Zhao L et al. LncRNA expression signatures of twist-induced epithelial-to-mesenchymal transition in MCF10A cells. Cell Signal 2014; 26 : 83-93.

47. Bonapace L, Coissieux MM, Wyckoff J, Mertz KD, Varga Z, Junt T et al. Cessation of CCL2 inhibition accelerates breast cancer metastasis by promoting angiogenesis. Nature 2014; 515: 130-133.

48. Shree T, Olson OC, Elie BT, Kester JC, Garfall AL, Simpson K et al. Macrophages and cathepsin proteases blunt chemotherapeutic response in breast cancer. Genes Dev 2011; 25: 2465-2479.

(c) (i) $(\&)$ This work is licensed under a Creative Commons Attribution-NonCommercial-NoDerivs 4.0 International License. The images or other third party material in this article are included in the article's Creative Commons license, unless indicated otherwise in the credit line; if the material is not included under the Creative Commons license, users will need to obtain permission from the license holder to reproduce the material. To view a copy of this license, visit http://creativecommons.org/licenses/by-nc-nd/4.0/ 